\title{
Carnosine quenches the reactive carbonyl acrolein in the central nervous system and attenuates autoimmune neuroinflammation
}

\author{
Jan Spaas ${ }^{1,2,3^{*}}$, Wouter M. A. Franssen ${ }^{2,4}$, Charly Keytsman 1,2,4 ${ }^{1}$ Laura Blancquaert ${ }^{3}$, Tim Vanmierlo ${ }^{1,5,6}$, \\ Jeroen Bogie ${ }^{1,2}$, Bieke Broux ${ }^{1,5,7}$, Niels Hellings ${ }^{1,5}$, Jack van Horssen ${ }^{1,2,8}$, Dheeraj Kumar Posa ${ }^{9}$, David Hoetker ${ }^{9}$, \\ Shahid P. Baba ${ }^{9}$, Wim Derave ${ }^{3 \dagger}$ and Bert O. Eijnde ${ }^{1,2 \dagger}$
}

\begin{abstract}
Background: Multiple sclerosis (MS) is a chronic autoimmune disease driven by sustained inflammation in the central nervous system. One of the pathological hallmarks of MS is extensive free radical production. However, the subsequent generation, potential pathological role, and detoxification of different lipid peroxidation-derived reactive carbonyl species during neuroinflammation are unclear, as are the therapeutic benefits of carbonyl quenchers. Here, we investigated the reactive carbonyl acrolein and (the therapeutic effect of) acrolein quenching by carnosine during neuroinflammation.

Methods: The abundance and localization of acrolein was investigated in inflammatory lesions of MS patients and experimental autoimmune encephalomyelitis (EAE) mice. In addition, we analysed carnosine levels and acrolein quenching by endogenous and exogenous carnosine in EAE. Finally, the therapeutic effect of exogenous carnosine was assessed in vivo (EAE) and in vitro (primary mouse microglia, macrophages, astrocytes).

Results: Acrolein was substantially increased in inflammatory lesions of MS patients and EAE mice. Levels of the dipeptide carnosine ( $\beta$-alanyl-L-histidine), an endogenous carbonyl quencher particularly reactive towards acrolein, and the carnosine-acrolein adduct (carnosine-propanal) were twofold lower within EAE spinal cord tissue. Oral carnosine treatment augmented spinal cord carnosine levels (up to > tenfold), increased carnosine-acrolein quenching, reduced acrolein-protein adduct formation, suppressed inflammatory activity, and alleviated clinical disease severity in EAE. In vivo and in vitro studies indicate that pro-inflammatory microglia/macrophages generate acrolein, which can be efficiently quenched by increasing carnosine availability, resulting in suppressed inflammatory activity. Other properties of carnosine (antioxidant, nitric oxide scavenging) may also contribute to the therapeutic effects.
\end{abstract}

Conclusions: Our results identify carbonyl (particularly acrolein) quenching by carnosine as a therapeutic strategy to counter inflammation and macromolecular damage in MS.

Keywords: Acrolein, Carnosine, Multiple sclerosis, Neuroinflammation, Oxidative stress, Reactive carbonyl

*Correspondence: jan.spaas@uhasselt.be

†Wim Derave and Bert O. Eijnde have equal contribution

${ }^{2}$ BIOMED Biomedical Research Institute, Faculty of Medicine and Life Sciences, Hasselt University, Hasselt, Belgium

Full list of author information is available at the end of the article

\section{Background}

In multiple sclerosis (MS), the infiltration of circulating lymphocytes and monocytes triggers sustained central nervous system (CNS) inflammation leading original author(s) and the source, provide a link to the Creative Commons licence, and indicate if changes were made. The images or other third party material in this article are included in the article's Creative Commons licence, unless indicated otherwise in a credit line to the material. If material is not included in the article's Creative Commons licence and your intended use is not permitted by statutory regulation or exceeds the permitted use, you will need to obtain permission directly from the copyright holder. To view a copy of this licence, visit http://creativecommons.org/licenses/by/4.0/. The Creative Commons Public Domain Dedication waiver (http://creativeco mmons.org/publicdomain/zero/1.0/) applies to the data made available in this article, unless otherwise stated in a credit line to the data. 
to demyelination and axonal damage [1-3]. Mounting evidence supports that reactive oxygen species (ROS) mediate CNS injury in both the acute and chronic disease stage $[4,5]$. The abundance of lipids renders the CNS extremely vulnerable to lipid peroxidation, a chain reaction of repetitive hydrogen abstractions from polyunsaturated fatty acids (PUFAs) resulting in the formation of reactive carbonyl species. Lipid-derived reactive carbonyls, including 4-hydroxy-2-nonenal (HNE) and acrolein, have a relatively long half-life and exert strong cytotoxic effects by covalently binding with surrounding nucleophilic macromolecules, i.e. proteins, RNA, DNA and lipids [6, 7]. In fact, it has become increasingly clear that reactive carbonyl species are not merely surrogate markers of oxidative injury but can mediate and aggravate disease progression by themselves. Accordingly, therapeutic strategies to prevent carbonyl (over)load are emerging for numerous disorders, such as diabetes/obesity $[8,9]$. Even though elevated levels of lipid-derived carbonyls have already been reported in MS [10-15], very little is known about the processes underlying their generation, potential pathological role, and detoxification, nor on the therapeutic benefits of carbonyl quenchers to counter CNS inflammation and macromolecular damage.

A variety of small molecules with the ability to quench and eliminate reactive carbonyl species are endogenously present in cells (phase II deactivation). The reactivity of different quenchers is dependent on the type of reactive carbonyl involved [16]. Carnosine ( $\beta$-alanyl-L-histidine) is a naturally occurring histidine-containing dipeptide (HCD) with carbonyl quenching properties linked to its amino group and imidazole ring [17, 18]. Alongside one or more related HCDs, such as homocarnosine, anserine or balenine, carnosine is predominantly found in excitable tissues (CNS and muscle), where it is synthetized by the enzyme carnosine synthase (CARNS1) [17, 19]. Carnosine is especially reactive towards $\alpha, \beta$-unsaturated carbonyls [6,20-22], of which acrolein is the strongest electrophile (approximately 100-fold more than HNE) [7, 23]. Molecular modelling studies and advances in detection techniques have yielded important insights in the formation and metabolism of carnosine-carbonyl adducts in vitro and in vivo $[8,16,24-26]$. Conjugation of carnosine to acrolein generates the oxidized product carnosine-propanal, which can be further reduced to carnosine-propanol before renal excretion [20]. By acting as a sacrificial nucleophile, exogenous carnosine administration has been suggested to slow the progression of oxidative-driven diseases $[17,18]$. The ability to cross the blood-brain barrier [27] and the favorable toxicological profile $[28,29]$ of this naturally occurring molecule underscore its therapeutic potential for CNS diseases.
Here, we studied the generation of acrolein during neuroinflammation and its quenching by endogenous and exogenous carnosine. Our data demonstrate significant acrolein-protein adduct formation in inflammatory lesions of MS patients and experimental autoimmune encephalomyelitis (EAE) mice, a model for autoimmune neuroinflammation that mimics MS. In EAE, this was associated with depletion of carnosine in spinal cord tissue, whereas oral carnosine intake augmented acrolein quenching and reduced inflammation and macromolecular damage. Our results identify carbonyl (in particular acrolein) quenching by carnosine as a therapeutic strategy for neuroinflammatory disorders such as MS.

\section{Materials and methods}

\section{Acrolein-protein adducts in active MS lesions}

Frozen brain material from active MS lesions was obtained from The Netherlands Brain Bank. Clinical patient details are depicted in Additional file 1: Table S1. Following acetone fixation (10 min) and blocking (30 min), serial cryosections were stained overnight for proteolipid protein (PLP) to assess demyelination and CD68 or HLA-DR to assess the presence and distribution of microglia/macrophages [30]. Staining was visualized with HRP-labelled secondary antibodies (Envision+, Dako) and 3,3'-Diaminobenzidine (DAB) substratechromogen (Dako). Similarly, sections were stained with antibodies against protein-bound acrolein $(1 / 100$, mouse monoclonal, 10A10, Novus Biologicals). For fluorescent double-staining, acrolein-protein adducts were visualized with secondary goat anti-mouse (1/600, AF 555, A-21425, Thermo Fisher), followed by overnight incubation with primary antibodies against CD68 (1/100, mouse monoclonal, M0814 KP1 clone, Dako) or glial fibrillary acidic protein (GFAP, 1/100, mouse monoclonal, G3893, Sigma), and secondary goat anti-mouse (1/400, IgG1 AF 488, A-21121, Thermo Fisher). Imaging was performed with a Leica DM4000 B LED (Leica Microsystems).

\section{Animals and EAE induction}

Female 9-to-11 week old C57BL/6 OlaHSD mice (Envigo) were actively immunized with two subcutaneous injections of $100 \mu \mathrm{L}$ myelin oligodendrocyte glycoprotein peptide fragment 35-55 emulsified in complete Freund's adjuvant $\left(\mathrm{MOG}_{35-55} / \mathrm{CFA}\right)$ in the upper and lower back. Intraperitoneal injections of $100 \mu \mathrm{L}$ pertussis toxin in phosphate-buffered saline (PBS) were administered immediately hereafter and $24 \mathrm{~h}$ later (EK-2110, Hooke Laboratories, MA). Clinical symptoms (scale $0-5$, including 0.5 increments: 0 , no symptoms; 1 , limp tail; 2, hindlimb paresis; 3 , hindlimb paralysis; 4 , front limb paresis; 5 , death due to EAE) and body weight (g) were monitored daily by a blinded assessor. Mice were 
housed on a $12 \mathrm{~h}: 12 \mathrm{~h}$ light:dark cycle under standard room conditions (temperature $20-24{ }^{\circ} \mathrm{C}$, relative humidity $30-60 \%$ ) with ad libitum access to drinking water and food pellets (Teklad 2018C, Envigo). In a separate experiment, carnosine treatment was investigated in a monophasic EAE model using female Lewis rats, as described previously (see Ref. [31] and Additional file 2: Table S2).

\section{EAE study procedures}

Healthy control and EAE mice were randomly allocated to different intervention groups. L-Carnosine treatment was administered by dissolving $3 \mathrm{~g} / \mathrm{L}$ (0.3\%), $15 \mathrm{~g} / \mathrm{L}$ (1.5\%) or $30 \mathrm{~g} / \mathrm{L}(3 \%)$ carnosine in the drinking water, starting 7 days prior to EAE induction until the end of the study period. Water bottles were refreshed every 2-3 days. L-Carnosine was a gift from Flamma (Flamma Group, Italy). EAE mice were sacrificed at the disease peak (acute EAE, days 14-18 post immunization), following partial recovery (subacute EAE, days 24-28) or in the chronic stage (day 56). Control mice were sacrificed along with the acute EAE mice. Following overdose injection of Dolethal (200 mg/kg, i.p.), blood was collected from the right ventricle with heparin-coated syringes. Mice were perfused with $\mathrm{PBS} /$ heparin $(25 \mathrm{UI} / \mathrm{mL})$ via a left ventricular puncture and spinal cords were isolated. Blood samples were centrifuged ( $5 \mathrm{~min}, 3500 \mathrm{rpm}$ ) and plasma was stored at $-80^{\circ} \mathrm{C}$. Spinal cords were cut in four segments that were placed immediately in liquid nitrogen or first embedded in Tissue-Tek O.C.T. compound, and stored at $-80{ }^{\circ} \mathrm{C}$. A graphical summary of the experimental designs used for the different analyses presented in this manuscript is shown in Additional file 4: Fig. S1.

\section{Detection of HCDs and carnosine-carbonyl adducts by UPLC-ESI-MS/MS}

Ultrahigh-performance liquid chromatography electrospray ionization tandem mass spectrometry (UPLCESI-MS/MS) was used to detect HCDs and their carbonyl conjugates. A 5\% homogenate was prepared from the thoracic spinal cord $(18.5 \pm 3.8 \mathrm{mg}$ wet tissue weight $(\mathrm{WW})$ ) in extraction solution containing $\mathrm{HCl}$ $(10 \mathrm{mM})$ and internal standards tyrosyl-histidine $(10 \mu \mathrm{M}$, Bachem), L-carnosine-d4 (10 $\mu \mathrm{M}, \mathrm{CDN}$ Isotopes) and L-anserine-d4 (10 $\mu \mathrm{M}, \mathrm{CDN}$ Isotopes). Following homogenisation (30 s, $3 \mathrm{~m} / \mathrm{s}$, Bead Ruptor Elite, Omni International), samples were sonicated on ice, centrifuged at $4{ }^{\circ} \mathrm{C}(10 \mathrm{~min}, 16,000 \times \mathrm{g})$, and supernatants were stored at $-80{ }^{\circ} \mathrm{C}$. Standards containing carnosine (1 mM, Sigma), anserine $(1 \mathrm{mM}$, Sigma) and homocarnosine $(1 \mathrm{mM}$, synthesized by Mike Wempe, PhD, University of Colorado) were prepared in extraction solution and were serially diluted (1:1) 19 times. Before analysis, standards and samples (spinal cord or plasma) were diluted $50 \times$ in a
75:25 acetonitrile:water mixture and vortexed. UPLCESI-MS/MS was performed by injecting $5 \mu \mathrm{L}$ sample into a Waters ACQUITY UPLC H-Class System coupled with a Xevo TQ-S micro triple quadrupole. The analytes were first separated by a Waters Acquity BEH HILIC column $(1.7 \mu \mathrm{m}, 2.1 \times 50 \mathrm{~mm})$ equipped with an in-line frit filter unit and then analysed by mass spectrometry in the positive mode. The analytes were eluted by using a binary solvent system consisting of $10 \mathrm{mM}$ ammonium formate, $0.125 \%$ formic acid in 5:95 acetonitrile:water (mobile phase A) or 95:5 acetonitrile:water (mobile phase B) at a flow rate of $0.55 \mathrm{~mL} / \mathrm{min}$. Initial conditions were 0.1:99.9 (A:B), ramping to $99.9: 0.1(\mathrm{~A}: \mathrm{B})$ over $5 \mathrm{~min}$ and then quickly ramping to 0.1:99.9 (A:B) over $0.5 \mathrm{~min}$.

Chromatograms were acquired using the transitions: carnosine $227 \rightarrow 110 \mathrm{~m} / z$, homocarnosine $241 \rightarrow 156 \mathrm{~m} / z$, anserine $241 \rightarrow 109 \mathrm{~m} / z$, carnosine-propanal $\quad 283 \rightarrow 166 \mathrm{~m} / z$, carnosine-propanol $285 \rightarrow 110 \mathrm{~m} / z$, carnosine-HNE $383 \rightarrow 110 \mathrm{~m} / z$, homocarnosine-acrolein $297 \rightarrow 110 \mathrm{~m} / z$, glutathione (GSH) $308 \rightarrow 179 \mathrm{~m} / z$, tyrosyl-histidine $319 \rightarrow 110 \mathrm{~m} / z$, carnosine-d4 $231 \rightarrow 110 \mathrm{~m} / z$, anserine- $\mathrm{d} 4245 \rightarrow 110 \mathrm{~m} / z$ in multiple reaction monitoring (MRM) mode. The AUC of carnosine, homocarnosine and carnosine-propanal were expressed relative to AUC of internal standard L-carnosine- $\mathrm{d} 4$, anserine relative to $\mathrm{L}$-anserine- $\mathrm{d} 4$ and GSH relative to tyrosyl-histidine. Analyte levels were calculated from the standard curve (carnosine, homocarnosine, anserine) or from the internal standard and expressed as nmol/mgWW. Sample protein content was determined to express analyte levels per $\mathrm{mg}$ protein (data available upon request). The lower limit of quantitation (LOQ) for carnosine was $24.4 \mathrm{nM}$, homocarnosine $48.9 \mathrm{nM}$, and anserine $24.4 \mathrm{nM}$. The coefficient of variation $(\mathrm{CV})$ of $\mathrm{HCD}$ measurements in spinal cord was determined using replicates within one sample run $(n=6)$ and between different sample runs $(n=3)$. Based on the calculation of total within-laboratory precision outlined by Chesher et al. [32], the $\mathrm{CV} \%$ of carnosine was $1.72 \%$, homocarnosine $3.01 \%$ and anserine $4.70 \%$.

\section{Acrolein- and HNE-protein adducts by western blot}

In order to quantify acrolein-protein and HNE-protein adducts by western blot, lumbrosacral spinal cord segments were manually homogenized on ice in RIPA buffer (50 mM Tris $\mathrm{pH} 8.0,150 \mathrm{mM} \mathrm{NaCl}, 0.5 \%$ sodium deoxycholate, $0.1 \%$ SDS, $1 \%$ Triton-X100) with freshly added protease and phosphatase inhibitors (Roche). Sample protein content was measured with Pierce BCA Protein Assay Kit (Thermo Fisher). Protein samples $(35 \mu \mathrm{g})$ were diluted in loading buffer and separated in $10 \%$ polyacrylamide gels. Next, proteins were transferred to PVDF membranes (90 min, $350 \mathrm{~mA}$ ) and blocked with 5\% milk 
(acrolein) or $5 \%$ bovine serum albumin (HNE) for $1 \mathrm{~h}$ at room temperature. Membranes were stained overnight at $4{ }^{\circ} \mathrm{C}$ in the appropriate blocking buffer using antibodies against protein-bound acrolein (1/2000, mouse monoclonal, 10A10, Novus Biologicals) or -HNE (1/1000, rabbit polyclonal, ab46545, Abcam). Secondary HRP-conjugated antibodies were applied for $90 \mathrm{~min}$ (1/3000, room temperature), followed by chemiluminescent detection with Pierce ECL Plus Western Blotting Substrate (Thermo Fisher) in the Amersham Imager 680 (Cytiva). Between different steps, membranes were washed in TBS-T. Ponceau S (Sigma) was used to stain all membrane proteins. Protein bands (a.u.) were quantified with ImageJ software and normalized to Ponceau S intensity at the same molecular weight.

\section{Quantitative polymerase chain reaction ( $q P C R$ )}

For gene expression analyses, total RNA was isolated from thoracic spinal cord segments using RNeasy Mini Kit (Qiagen). Briefly, samples were homogenized in $1 \mathrm{~mL}$ QIAzol reagent (Qiagen), mixed with chloroform and centrifuged (15 min, $12,000 \mathrm{rpm}, 4{ }^{\circ} \mathrm{C}$ ). The RNA layer was diluted in $70 \%$ ethanol and spinned down multiple times in the spin columns according to manufacturer's instructions. Final RNA was diluted in RNAse-free water and concentration was measured with a NanoDrop spectrophotometer (Thermo Fisher) at $260 \mathrm{~nm}$. Next, cDNA with a final concentration of $5 \mathrm{ng} / \mu \mathrm{L}$ was synthesized using 1000 ng RNA and qScript cDNA SuperMix (Quantabio). Real-time quantitative PCR (qPCR) was conducted on a StepOnePlus detection system (Applied Biosystems) using Fast SYBR Green Master Mix (Applied Biosystems). Primer sequences are detailed in Additional file 3: Table S3. The most stable reference genes were used for normalisation (geNorm) [33]. Analysis was performed with the $2^{-\Delta \Delta \mathrm{Ct}}$ method and expressed as fold changes.

\section{Immunohistochemistry}

Thoracolumbar spinal cord cryosections $(10 \mu \mathrm{m})$ were used to stain $\mathrm{T}$ cells (CD3), microglia/macrophages (F4/80), astrocytes (GFAP), and acrolein-protein adducts. After acetone fixation (10 $\mathrm{min})$ and blocking (30 $\mathrm{min})$, sections were incubated with primary antibodies detecting F4/80 (1/100, overnight, rat monoclonal, MCA497G, Bio-Rad), CD3 (1/150, overnight, rat monoclonal, MCA500G, Bio-Rad), or GFAP (1/100, 3 h, mouse monoclonal, G3893, Sigma), followed by multiple washes and complementary secondary antibodies for $1 \mathrm{~h}$ (Thermo Fisher). Using fluorescent microscopy (Leica DM4000 B LED, Leica Microsystems), the entire spinal cord section was captured in a series of images. The total number of $\mathrm{T}$ cells or microglia/macrophages was calculated by dividing the entire positive stained area by the average area of one cell, and expressed per $\mathrm{mm}^{2}$ tissue (ImageJ) [34]. For astrocytes, the percentage $\mathrm{GFAP}^{+}$area vs. total area was used (ImageJ). In a subset of experiments, acrolein-protein adducts (1/100, mouse monoclonal, 10A10, Novus Biologicals) were co-stained with F4/80 or GFAP. The acrolein-positive area (\%) was analysed using ImageJ in entire spinal cord sections, and in lesion vs. non-lesion areas separately. Lesions were determined by hypercellularity (DAPI nuclear stain) and accumulation of $\mathrm{F} 4 / 80^{+}$ cells. Negative control sections omitting one or all primary antibodies were included for all immunostainings.

\section{Cell culture}

Primary microglial cell cultures were prepared from newborn (P0-2) C57BL/6 mouse pups. Briefly, brain cortices were freed from meninges, kept on ice, homogenised and dissociated to single-cell suspension with papain (20 U/ $\mathrm{mL}$, Sigma) and DNAse $(0.15 \mathrm{mg} / \mathrm{mL}$, Sigma $)$. Mixed glial cell cultures were grown in PLL-coated flasks with DMEM D6429 (Sigma), 10\% heat-inactivated fetal bovine serum (FBS, Biowest), 1\% Penicillin-Streptomycin (PS, Sigma), at $37{ }^{\circ} \mathrm{C}, 5 \% \mathrm{CO}_{2}$. On days 3,7 and 11 after isolation, cell medium was fully replaced. Starting from day 3, L929 cell-conditioned medium (LCM) was added in a 1:2 ratio to promote microglial proliferation. On day 14 , microglia were collected after shake-off procedure $(230 \mathrm{rpm}, 4 \mathrm{~h})$, centrifuged $(10 \mathrm{~min}, 300 \times g)$ and plated in DMEM containing 15\% LCM. After shake-off, astrocytes were purified from mixed glial cultures over a period of 7-10 days by differential adhesion and mild trypsinization (Sigma). Mouse bone marrow-derived macrophages (BMDMs) were obtained from femoral and tibial bone marrow suspensions of 11-week-old C57BL/6 mice. BMDMs were differentiated for 7 days in RPMI 1640 medium (Lonza Bioscience) supplemented with 10\% FBS, $0.5 \%$ PS, and 15\% LCM (5\% after plating) [35].

In all experiments, pre-treatment with L-carnosine (Flamma), L-anserine (Flamma), L-balenine (NNB Nutrition), $\beta$-alanine (Sigma), L-histidine (Sigma), tyrosyl-histidine (Bachem) or vehicle control (PBS) was given for $24 \mathrm{~h}$ (1-20 mM). Hereafter, cell medium was refreshed and cells were exposed to either lipopolysaccharide (LPS, $100 \mathrm{ng} / \mathrm{mL}, 24 \mathrm{~h}$, Merck), hydrogen peroxide $\left(\mathrm{H}_{2} \mathrm{O}_{2}\right.$, $100 \mu \mathrm{M}, 24 \mathrm{~h}, \mathrm{VWR}$ Chemicals), phorbol 12-myristate 13-acetate (PMA, $100 \mathrm{ng} / \mathrm{mL}, 15 \mathrm{~min}$, Sigma), recombinant murine IFN- $\gamma$ and IL- $1 \beta(100 \mathrm{ng} / \mathrm{mL}$ and $10 \mathrm{ng} / \mathrm{mL}$, $24 \mathrm{~h}$, PreproTech), or vehicle control (PBS). For determination of HCDs and their carbonyl conjugates (2e6 cells/ flask), cells were washed, scraped, collected, centrifuged and stored at $-80{ }^{\circ} \mathrm{C}$. Cell pellets were resuspended in extraction solution and processed for UPLC-ESI-MS/ MS as described above. For gene expression analysis 
following LPS exposure (2e5 cells/well), cells were lysed in QIAzol (Qiagen), followed by RNA extraction, cDNA synthesis and qPCR amplification as described above. For flow cytometry (1.5e 5 cells/well), LPS-stimulated cells were detached in PBS/EDTA (10 mM, $10 \mathrm{~min}$ ), centrifuged, and stained with Fixable Viability Dye (FVD) eFluor 506 (1/1000, eBioscience) for $30 \mathrm{~min}$ at $4{ }^{\circ} \mathrm{C}$. Next, cells were stained by F4/80-PeCy7 (1/100, Biolegend) and MHC class II-PE (1/500, eBioscience) for $15 \mathrm{~min}$ at room temperature. Flow cytometry was run on a BD LSRFortessa. Using FlowJo software (LLC, BD), the MHC-II mean fluorescence intensity was determined from the single cell, $\mathrm{FVD}^{-}, \mathrm{F} 4 / 80^{+}$population. To determine intracellular ROS levels (5e4 cells/well), PMA-stimulated cells were incubated with $2^{\prime}, 7^{\prime}$-Dichlorofluorescin diacetate (DCFH-DA, Sigma) for $30 \mathrm{~min}$. Fluorescence spectroscopy detection with excitation/emission at $485 / 520 \mathrm{~nm}$ was performed immediately to measure the oxidized product 2',7'-dichlorofluorescein (FLUOstar Optima, BMG Labtech). To determine nitrite release, an indicator of cellular nitric oxide production, culture medium from $1 \mathrm{e} 5$ cells $/ 100 \mu \mathrm{L}$ was obtained after LPS or IFN- $\gamma /$ IL-1 $\beta$ exposure and 1:1 diluted with a mixture containing $\mathrm{N}$-(1-Naphthyl)ethylenediamine dihydrochloride (Sigma) and Sulfanilamide (Sigma). The absorbance of the reaction product was read at $540 \mathrm{~nm}$ (iMark, Bio-Rad) and the concentration $(\mu \mathrm{M})$ of nitrite was calculated from a linear standard curve containing Sodium Nitrite (Promega). All in vitro experiments were repeated $\geq 3$ times.

\section{Statistics}

Statistical analyses were performed with IBM SPSS Statistics v25 (NY, USA). Two independent groups were compared by $\mathrm{t}$-tests or Mann-Whitney $\mathrm{U}$ tests, with Bonferroni-adjusted $\mathrm{p}$ values in case of multiple testing. Multiple groups were compared by one-way ANOVA and Tukey HSD post hoc tests, or Kruskal-Wallis with Bonferroni-adjusted pairwise comparisons. The timedependent effect of carnosine treatment (Fig. 4) was analysed by two-way ANOVA (group $\times$ treatment, $4 \times 2$ ), followed by Bonferroni-corrected pairwise t-testing (per group) and one-way ANOVA (delta treatment values). AUC of clinical EAE scores was calculated in GraphPad Prism v7.04. Data are presented as mean \pm SD, unless otherwise specified. " $\mathrm{p}<0.05$, "* $\mathrm{p}<0.01$ for two-sided hypothesis testing.

\section{Results \\ Acrolein-protein adduct formation in active MS lesions and EAE infiltrates}

Excessive free radical production is a pathological hallmark of CNS inflammation in MS. To evaluate the downstream generation of acrolein, a highly reactive lipid-derived carbonyl that can induce significant protein modification [6], we examined acrolein-protein adduct formation in human brain samples containing demyelinated $\left(\mathrm{PLP}^{-}\right.$) active MS lesions (with microglia/macrophages throughout the lesion area) and mixed active/ inactive MS lesions (hypocellular lesion center with microglia/macrophages at the border) by immunohistochemistry [30]. Acrolein-protein adducts were present in both active and mixed active/inactive lesions (Fig. 1a, b), yet in some mixed active/inactive lesions no acroleinprotein immunoreactivity could be visualized via $D A B$ staining (Fig. 1c). Notably, the pattern of acrolein staining was not uniform and varied between tissue blocks and between different areas of the same tissue block. In certain lesions, we observed acrolein-protein adducts surrounding densely packed $\mathrm{CD}^{+} 8^{+}$microglia/macrophages (Fig. 1d) or in perivascular cuffs (Fig. 1e). In addition, acrolein-protein adducts displaying a specific cellular morphology were repeatedly observed in the lesion center, lesion border, or even outside of the lesion $\left(\mathrm{PLP}^{+} / \mathrm{CD}^{-} 8^{-}\right.$ area). These cellular structures were identified as $\mathrm{GFAP}^{+}$ reactive astrocytes (Fig. 1f) and were $\mathrm{CD}^{-} 8^{-}$(Fig. 1g).

We next examined whether acrolein- and HNE-protein adducts are increased within the spinal cord of EAE mice; an animal MS model featuring autoimmune neuroinflammation that is triggered by immunization with myelin antigens leading to lymphocyte and monocyte infiltration into the CNS [36]. Using a 0-5 clinical disability grading system (see methods), EAE mice displayed an initial disease peak (acute EAE, days 14-18 post immunization, clinical disability score $2.5 \pm 1.1$ ), followed by a slight remission (subacute EAE, days 24-28, score 1.9 \pm 0.9 ) and stabilization (chronic EAE, day 56, score $2.2 \pm 0.3$ ). Spinal cord mRNA expression profiles (Additional file 4: Figure S2a) and accumulation of $\mathrm{F} 4 / 80^{+}$microglia/macrophages in spinal cord sections (Additional file 4: Fig. S2b) confirmed higher levels of inflammatory activity and oxidative/nitrosative stress in acute compared to chronic EAE. When examining spinal cord acroleinprotein adduct formation by western blot in EAE vs. control mice, increased acrolein-protein adducts were found at multiple molecular weights $(25,50,55,60 \mathrm{kDa})$ and at all EAE disease stages, with acute EAE mice exhibiting the greatest increase in acrolein damage (Fig. 2a). To further study the location of these adducts within the spinal cord, immunohistochemistry was performed and revealed that acrolein-protein adduct formation was predominantly present in inflammatory lesions in proximity to $\mathrm{F} 4 / 80^{+}$microglia and macrophages (Fig. $2 \mathrm{~b}$, c); both in the parenchyma and in perivascular cuffs (Fig. 2d, e). The fact that most acrolein immunoreactivity was found in or around such infiltrates suggests that the oxidative burst from inflammatory microglia/macrophages is a major 


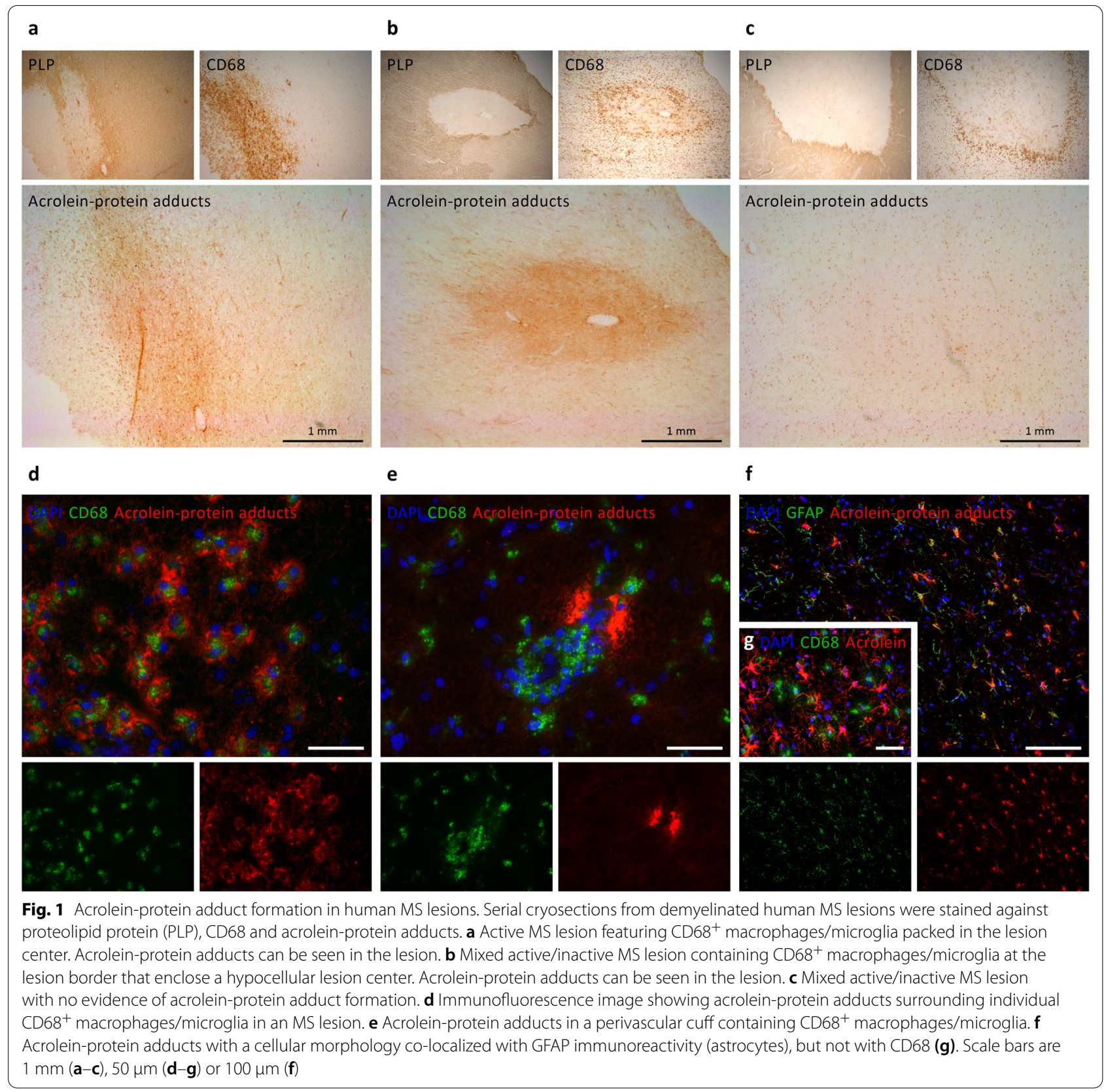

source of acrolein accumulation in EAE spinal cord. Acrolein-protein adducts were also observed in structures with morphological features resembling astrocytic processes that were $\mathrm{GFAP}^{+}$(Fig. 2f) but F4/80- (Fig. 2e). We also examined whether the formation of HNE-protein adducts was increased in EAE spinal cords, but HNEmodified proteins were unaffected compared to control mice by western blot analysis (Additional file 4: Figure S3). Taken together, active MS lesions and inflammatory EAE infiltrates are featured by substantial levels of acrolein-protein adducts. These adducts were localized in and in close proximity to inflammatory microglia/macrophages, but also occurred in other structures such as astrocytes.

\section{Carnosine quenches acrolein in spinal cord but is depleted in $\mathrm{EAE}$}

Several endogenous quenchers and enzymes, which form conjugates and metabolize reactive carbonyls, are present in the CNS $[20,37,38]$. Here, we studied the histidine-containing dipeptides (HCDs), which exhibit high quenching activity towards acrolein $[16,22]$. To 


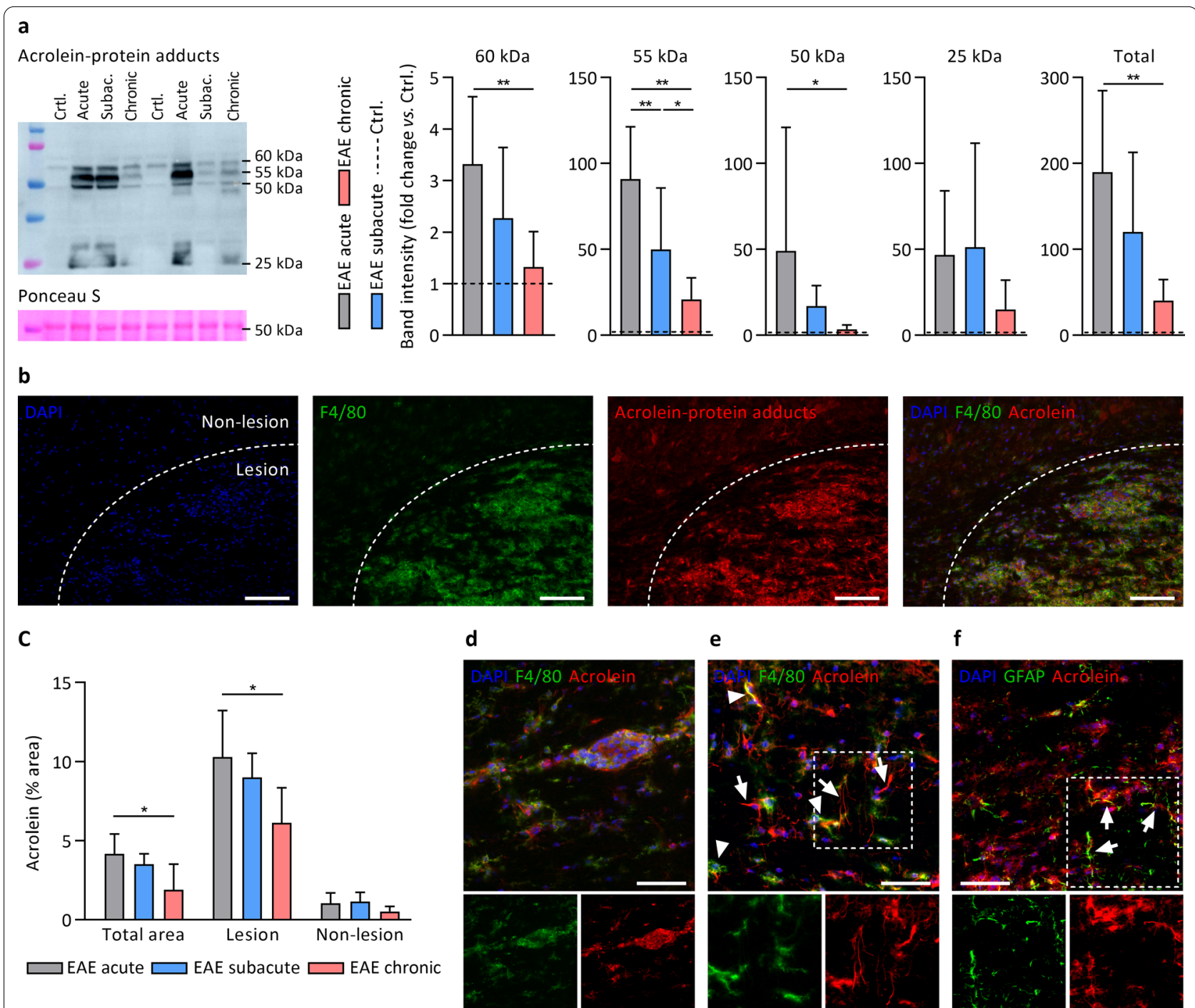

Fig. 2 Acrolein-protein adduct formation coincides with high levels of inflammation and microglia/macrophage accumulation in different stages of EAE. a Detection of acrolein-protein adducts by western blot in different stages of EAE compared to healthy controls (dashed line). $n=7-8$ animals/group. Acrolein-protein adducts were increased in EAE vs. healthy controls (statistics not shown), except for subacute and chronic EAE at $60 \mathrm{kDa}$, and chronic EAE at $50 \mathrm{kDa}$. $\mathbf{b}$ Immunohistochemistry demonstrating acrolein-protein adducts in hypercellular EAE spinal cord lesions characterized by an abundance of $\mathrm{F} 4 / 80^{+}$microglia/macrophages. c Quantification of acrolein-protein adduct immunoreactivity (\% positive vs. total area) in EAE spinal cord sections, showing higher acrolein-protein levels in lesion vs. non-lesion areas, and in acute vs. chronic EAE. $n=6$ animals/ group. $\mathbf{d}$ Acrolein-protein adducts in perivascular cuff containing $\mathrm{F} 4 / 80^{+}$microglia/macrophages. e Acrolein-protein adducts in brain parenchyma co-localized with F4/80+ microglia/macrophages (arrowheads) and with astrocytic processes that appeared F4/80- (arrows), but GFAP (arrows in f). Data are mean \pm SD. One-way ANOVA or Kruskal-Wallis, post hoc testing ${ }^{*} p<0.05,{ }^{* *} p<0.01$ between the indicated groups. Scale bars are $100 \mu \mathrm{m}(\mathbf{b})$ or $50 \mu \mathrm{m}(\mathbf{d}-\mathbf{f})$

determine whether the levels of these dipeptides and their carbonyl conjugates are affected within the spinal cord during different stages of EAE, an extensive profiling using UPLC-ESI-MS/MS was performed. The HCDs carnosine $(0.23 \pm 0.03 \mathrm{nmol} / \mathrm{mgWW})$, homocarnosine $(0.50 \pm 0.06 \mathrm{nmol} / \mathrm{mgWW})$ and anserine $(0.016 \pm 0.006 \mathrm{nmol} / \mathrm{mgWW})$ were detected in mouse spinal cord, in addition to carnosine-propanal; the oxidized form of a carnosine-acrolein adduct $(0.0018 \pm 0.0002 \mathrm{nmol} / \mathrm{mgWW})$. During acute EAE, carnosine (Fig. 3a) and carnosine-propanal (Fig. 3b) dropped to $0.12 \pm 0.04 \mathrm{nmol} / \mathrm{mgWW}(-46 \%)$ and $0.0010 \pm 0.0003 \mathrm{nmol} / \mathrm{mgWW} \quad(-44 \%)$ respectively, before gradually recovering; indicating that carnosine homeostasis is challenged during EAE. Carnosine and carnosine-propanal levels strongly correlated with each 
a

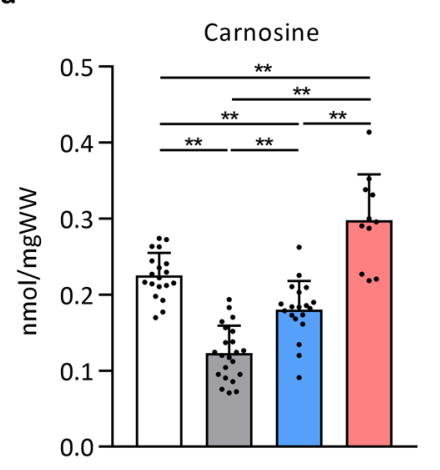

d

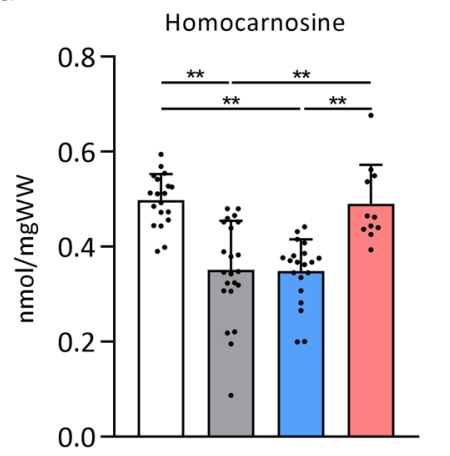

b
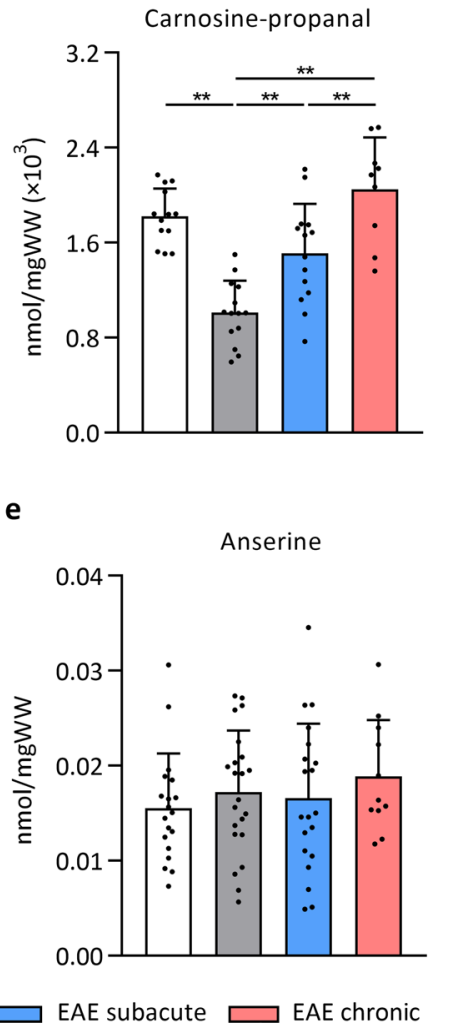

C

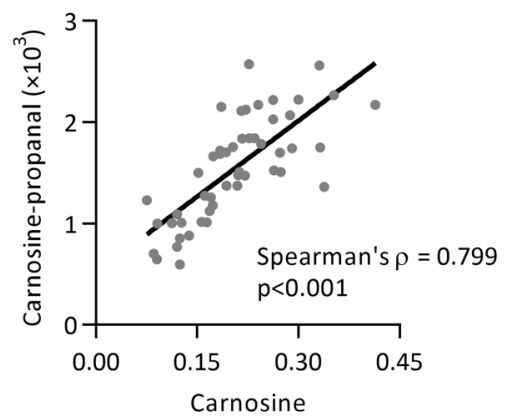

f

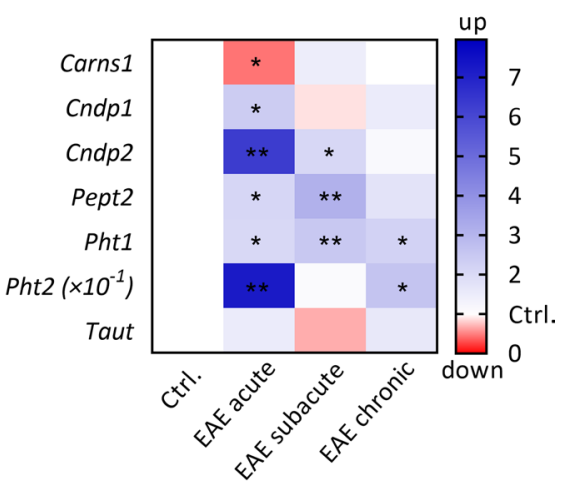

Fig. 3 Spinal cord carnosine and carnosine-propanal are depleted in acute EAE. UPLC-ESI-MS/MS-based quantification of histidine-containing dipeptides and their carbonyl conjugates shows changes in $\mathbf{a}, \mathbf{c}$ carnosine, $\mathbf{b}, \mathbf{c}$ carnosine-propanal and $\mathbf{d}$ homocarnosine, but not $\mathbf{e}$ anserine, in different stages of EAE compared to healthy control mice. $n=8-23$ animals/group. Carnosine-propanal values $(\sim 0.0015 \mathrm{nmol} / \mathrm{mgWW})$ were transformed $\left(\times 10^{3}\right)$ to facilitate visualisation on the $Y$ axis. $\mathbf{f} \mathrm{mRNA}$ expression of key regulators involved in carnosine homeostasis was altered in EAE spinal cord compared to healthy controls. Gene expression data are fold changes vs. control mice. Due to high expression of Pht2 in EAE mice, data were transformed $\left(\times 10^{-1}\right)$ to allow visualisation. $n=6-7$ animals/group. Carns1, carnosine synthase; Cndp 1, carnosine dipeptidase 1; Cndp2, cytosolic non-specific dipeptidase 2; Pept2, peptide transporter 2 (Slc15a2); Pht1, peptide/histidine transporter 1 (Slc15a4); Pht2, peptide/histidine transporter 2 (SIc15a3); Taut, taurine transporter (SIc6a6). Data are mean \pm SD. One-way ANOVA or Kruskal-Wallis, post hoc testing * $p<0.05$, ${ }^{* *} \mathrm{p}<0.01$ between the indicated groups $(\mathbf{a}, \mathbf{b}, \mathbf{d}, \mathbf{e})$ or vs. healthy control mice $(\mathbf{f})$

other (Fig. 3c). Homocarnosine levels also decreased in acute and subacute $\operatorname{EAE}(-30 \%$, Fig. 3d), whereas anserine remained unchanged (Fig. 3e). No carnosinepropanol, carnosine-HNE or homocarnosine-acrolein conjugates were detected either in the healthy or EAE mice. Similar to carnosine, the levels of the endogenous antioxidant and carbonyl quencher GSH were decreased in (sub)acute EAE (-30\%, Additional file 4: Fig. S4).

HCD homeostasis is maintained by a complex interplay between synthesis, breakdown and transmembrane transport [17]. To gain insight in the mechanism by which the HCDs are depleted within the spinal cord of EAE mice, we next determined the spinal cord gene expression of key regulators involved in carnosine synthesis (Carns1), breakdown (Cndp1, Cndp2) and transport (Pept2, Pht1, Pht2, Taut). As shown in Fig. 3f,
Carns1 expression decreased $(-52 \%)$ in acute EAE, whilst the expression of carnosine-hydrolysing enzymes and transporters remained generally elevated throughout the disease course. Collectively, these data indicate that carnosine quenches acrolein to form carnosine-propanal in mouse spinal cord, and that endogenous carnosine homeostasis is disturbed during EAE, which is potentially linked to excessive acrolein load or alterations in the synthesis and breakdown of the dipeptide.

\section{Carnosine treatment increases carnosine and carnosine-propanal levels in a time-dependent manner in EAE spinal cord tissue}

Given that the spinal cord carnosine levels were decreased in EAE mice, we next studied if exogenous (oral) carnosine intake can increase the availability of 
carnosine in spinal cord tissue of mice. For this purpose, control and EAE mice were treated with and without $1.5 \%$ L-carnosine in drinking water (from 7 days before immunization). Carnosine treatment significantly increased spinal cord carnosine levels in both healthy and EAE mice (Fig. 4a). Notably, the magnitude of carnosine increase was strongly influenced by the EAE disease stage, with acute EAE mice displaying the greatest effect (absolute increase of $1.68 \pm 0.49 \mathrm{nmol} / \mathrm{mgWW}$ ) despite a shorter total treatment duration than the subacute and chronic EAE mice. In carnosine-treated control and chronic EAE mice, which displayed the smallest effect, substantial carnosine loading was still apparent in spinal cord (change compared to untreated reference group: control $+175 \%$, acute $\mathrm{EAE}+1363 \%$, subacute $\mathrm{EAE}+481 \%$, chronic $\mathrm{EAE}+126 \%)$. To determine whether increasing carnosine availability could affect the conjugation or removal of reactive carbonyls, we also examined the levels of the carnosine-carbonyl conjugates and found that the formation of carnosine-propanal within the spinal cord was significantly increased in a similar time-dependent manner (compared to untreated reference group: control $+23 \%$, acute $\mathrm{EAE}+268 \%$, subacute $\mathrm{EAE}+103 \%$, chronic $\mathrm{EAE}+36 \%$, Fig. $4 \mathrm{~b})$. In addition, small levels of carnosine-propanol (the reduced carnosine-acrolein adduct) were detectable in mice receiving oral carnosine,

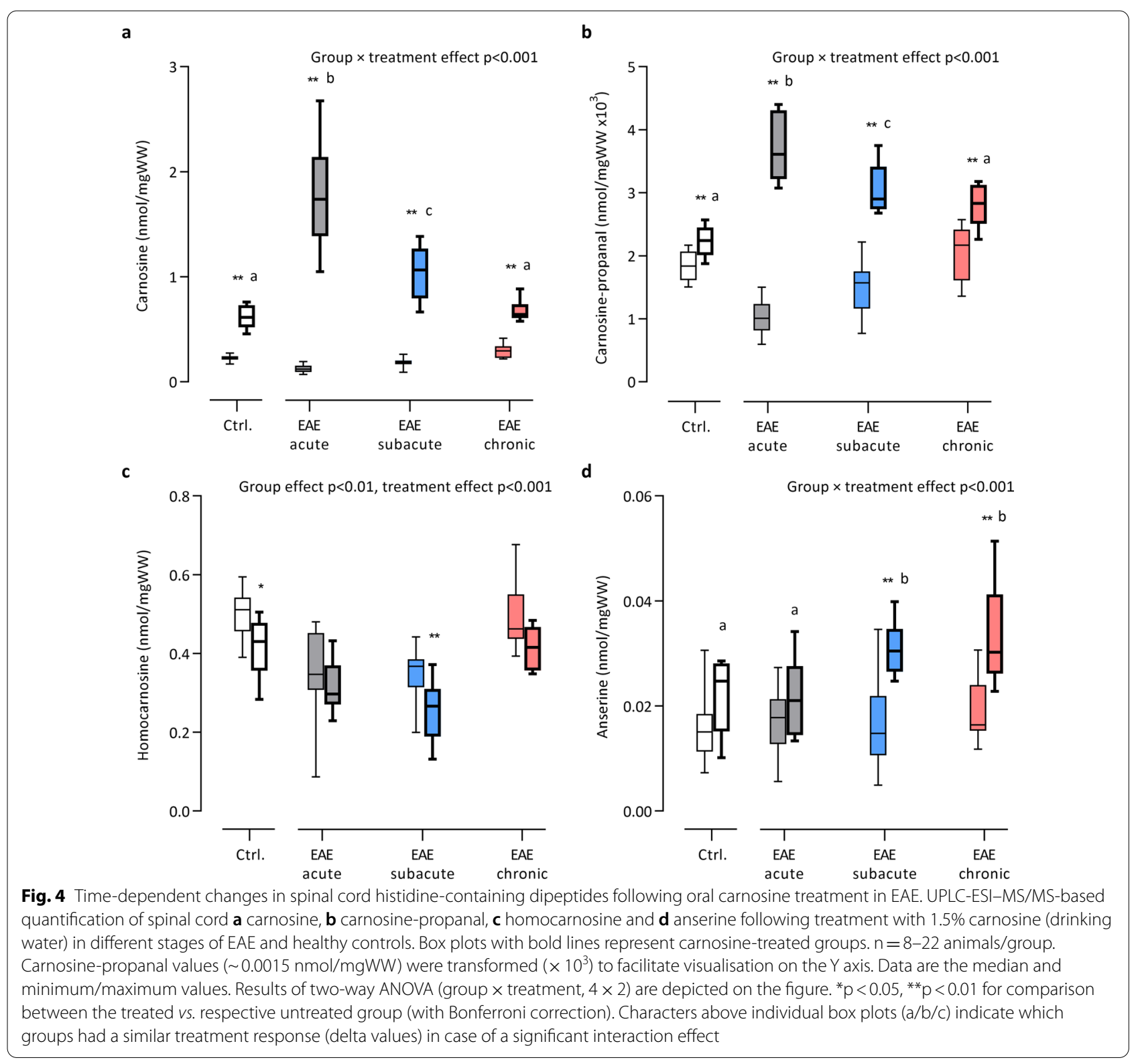


but could not be quantified. Homocarnosine was slightly reduced by carnosine treatment (Fig. 4c). Long-term (28 and 56 days) carnosine treatment significantly increased anserine levels in EAE spinal cord (Fig. 4d). In conclusion, these findings show that exogenous carnosine abundantly reaches the spinal cord to enhance local carnosine levels and promote acrolein quenching. In EAE, these effects are most pronounced in the acute stage.

\section{Carnosine treatment dose-dependently attenuates EAE disease severity}

To investigate the therapeutic potential of carnosine treatment and whether carnosine enhances acrolein quenching in EAE, mice were treated with 3 different carnosine doses $(0.3 \%, 1.5 \%$ or $3 \%$ in drinking water) and scored daily on a $0-5$ scale for paresis assessment. To ensure dose-dependent carnosine loading, carnosine levels within the spinal cord isolated from the chronic stage were analysed. Compared to untreated EAE mice $(0.30 \pm 0.06 \mathrm{nmol} / \mathrm{mgWW})$, carnosine levels were increased in the groups receiving $1.5 \%$ carnosine $(0.68 \pm 0.10 \mathrm{nmol} / \mathrm{mgWW},+126 \%)$ and $3 \%$ carnosine $(1.32 \pm 0.38 \mathrm{nmol} / \mathrm{mgWW},+343 \%$, Fig. $5 \mathrm{a})$. As expected, increased carnosine availability also increased acrolein quenching, with carnosine-propanal displaying a similar dose-dependent pattern in the $1.5 \%$ and $3 \%$ carnosine groups $(0.0028 \pm 0.0003 \mathrm{nmol} / \mathrm{mgWW},+36 \%$ and $0.0039 \pm 0.0004 \mathrm{nmol} / \mathrm{mgWW},+89 \%$, respectively) compared to untreated EAE mice $(0.0020 \pm 0.0004 \mathrm{nmol} /$ mgWW, Fig. 5b). Carnosine-propanol was detectable, yet not quantifiable, in spinal cord of EAE mice receiving either $1.5 \%$ or $3 \%$ carnosine. Dose-dependent increases in carnosine and carnosine-propanal were also observed in plasma (Fig. 5c, d). Homocarnosine, anserine and GSH levels are presented in Additional file 4: Figure S5.

EAE disease severity was attenuated by carnosine treatment. The clinical effect mainly occurred after the acute stage (Fig. 6a), and the cumulative clinical disability (area under the curve, AUC) was significantly lower in mice receiving $3 \%$ carnosine (AUC $68.9 \pm 13.7$ ) compared to all other groups (EAE, 85.0 \pm 14.4 ; EAE 0.3\%, $84.0 \pm 14.4$; EAE 1.5\%, 82.9 \pm 15.1 ; all $\mathrm{p}<0.05)$. No clinical effect occurred at disease onset or peak. Mice were sacrificed 56 days post immunization. By western blot, we defined whether carnosine treatment was able to limit acrolein-protein adduct formation. As shown in Fig. 6b, carnosine treatment markedly reduced acrolein-protein adduct levels, by $\sim 50 \%$ for proteins of 50 and $55 \mathrm{kDa}$ and by $\sim 25 \%$ overall. In contrast, HNE-protein adduct levels were unaffected by EAE and carnosine (Additional file 4: Fig. S6). The beneficial effect of carnosine on EAE disease severity was confirmed by immunohistochemistry showing reduced $\mathrm{CD}^{+} \mathrm{T}$ cell and $\mathrm{F} 4 / 80^{+}$microglia and macrophage numbers in spinal cord (Fig. 6c-f). Carnosine did not impact GFAP $^{+}$reactive astrocytes in spinal cord (Fig. 6g, h). Gene expression analysis revealed a dose-dependent reduction in inflammatory cytokines, Nos 2 and H2-DMa (MHC-II) mRNA levels following carnosine treatment (Fig. 6i). Finally, we aimed to replicate the protective effect of oral carnosine treatment $(1.5 \%$ in drinking water) in a different species (rat) and EAE model (monophasic spontaneously recovering disease course, 17 days), and we could confirm a $30 \%$ reduction $(\mathrm{p}<0.01)$ in EAE cumulative clinical disability (see details in Additional file 2: Table S2). Taken together, carnosine treatment dose-dependently attenuated clinical disease severity and markers of neuroinflammation in EAE. The effects are accompanied by increased acrolein quenching and reduced acrolein-protein adduct formation in spinal cord using high-dose carnosine treatment.

\section{Carnosine quenches inflammation-induced acrolein and modulates microglia and macrophage inflammation in vitro}

Given that acrolein generation in MS and EAE lesions spatially and temporally coincided with pro-inflammatory microglia/macrophages, and that acrolein quenching by carnosine suppressed neuroinflammation in EAE, we next used primary mouse microglial cell cultures to study acrolein generation and a potential direct anti-inflammatory effect of carnosine (Fig. 7a). First, UPLC-ESI-MS/ MS analyses were performed to assess carnosine levels and acrolein quenching in microglia after exposure to carnosine $(10 \mathrm{mM}, 24 \mathrm{~h})$ followed by LPS or $\mathrm{H}_{2} \mathrm{O}_{2}$ treatment. Microglia contained low basal levels of carnosine ( $\sim 0.025 \mathrm{nmol} / 1 \mathrm{e} 6$ cells), which rose approximately 50 - to 100-fold upon carnosine exposure (Fig. 7b). Interestingly, carnosine-propanal adducts were detected in carnosinetreated microglia under inflammatory conditions (TLR4 activation by LPS) or, to a lesser extent, following direct exposure to an oxidant $\left(\mathrm{H}_{2} \mathrm{O}_{2}\right)$; illustrating both the acrolein-generating ability of inflammatory microglia and the quenching by carnosine (Fig. $7 \mathrm{c}$ ). Carnosine and carnosine-propanal correlated with each other (Additional file 4: Fig. S7a), and when expressed per mg protein, carnosine and carnosine-propanal levels were within the same range in vitro (microglia) and in vivo (EAE spinal cord) (data not shown). Furthermore, low levels of anserine were observed in carnosine-treated cells (Additional file 4: Fig. S7b), whilst no homocarnosine, carnosine-propanol or carnosine-HNE was present. Cell viability was unaffected under all experimental conditions (Additional file 4: Fig. S7c).

To study if carnosine treatment directly affects inflammatory activity of microglia, microglial cultures were pre-treated with carnosine $(10 \mathrm{mM}, 24 \mathrm{~h})$, 


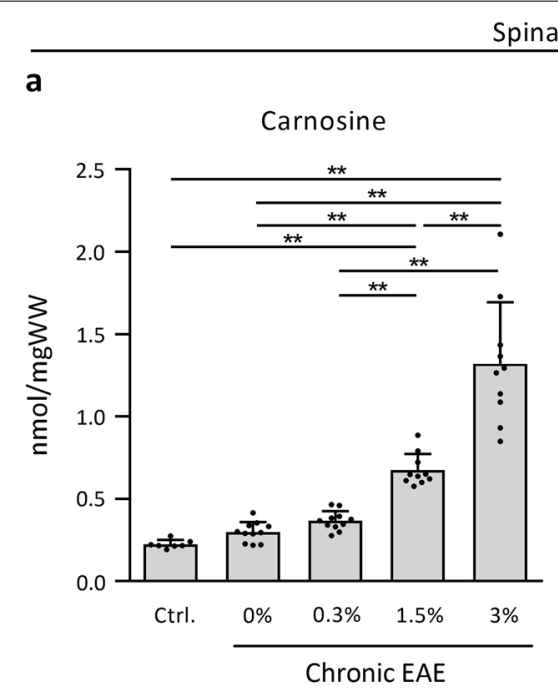

b

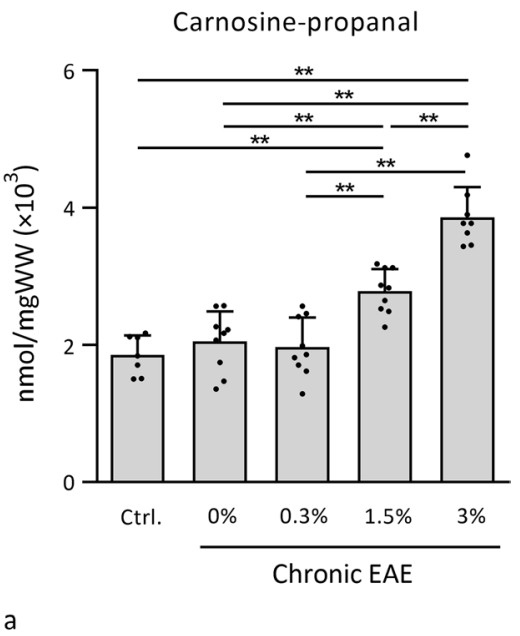

c

d
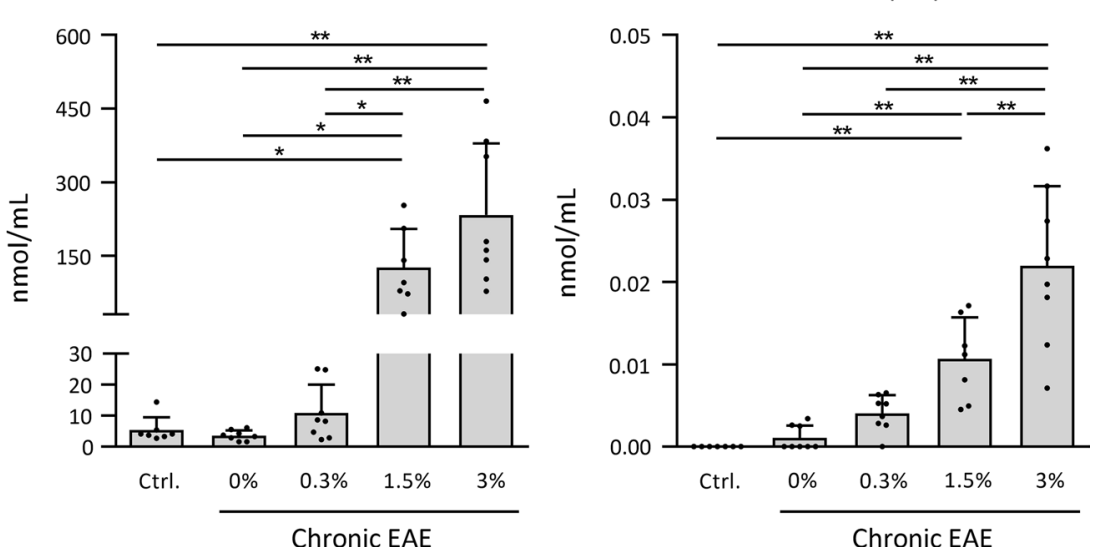

Fig. 5 Carnosine treatment dose-dependently increases carnosine and carnosine-propanal in chronic EAE. UPLC-ESI-MS/MS-based quantification of spinal cord and plasma a, c carnosine and $\mathbf{b}$, $\mathbf{d}$ carnosine-propanal in healthy controls, untreated EAE animals (0\%) and following treatment with different doses of carnosine in drinking water $(0.3 \%, 1.5 \%, 3 \%)$ in EAE. In figure $\mathbf{d}$, carnosine-propanal was not detected in $7 / 7 \mathrm{control}$ mice, 5/8 untreated EAE mice, and 1/8 mice receiving 0.3\% carnosine. $\mathrm{n}=7-11$ animals/group. Carnosine-propanal values $(\sim 0.0015 \mathrm{nmol} / \mathrm{mgWW})$ were transformed $\left(\times 10^{3}\right)$ to facilitate visualisation on the Y axis. Data are mean \pm SD. One-way ANOVA or Kruskal-Wallis, post hoc testing ${ }^{*} p<0.05$, ${ }^{* *} p<0.01$ between the indicated groups

exposed to an inflammatory trigger, and subsequently analysed for several functional/inflammatory indicators. We observed that carnosine suppressed gene expression of pro-inflammatory cytokines, Nos 2 and H2-DMa (MHC-II) following LPS exposure for $24 \mathrm{~h}$ (Fig. 7d) but not $6 \mathrm{~h}$ (data not shown). Consistent with these findings, flow cytometric analyses demonstrated that carnosine completely suppressed the LPS-induced MHC-II surface expression (Fig. 7e). Similarly, carnosine pre-treatment dose-dependently reduced microglial ROS levels (Fig. 7f) and nitrite release, an indicator of nitric oxide production (Fig. $7 \mathrm{~g}$ ). Carnosine did not affect basal nitrite release by naive microglia (data not shown). Suppressed nitrite release was confirmed, even at lower doses of carnosine, when using IFN- $\gamma /$ IL-1 $\beta$ to activate microglia (Fig. $7 \mathrm{~h}$ ). We next investigated different cell populations relevant to neuroinflammation in MS/EAE, and found that carnosine also reduced nitrite release from mouse BMDMs (Fig. 7i), but not primary mouse astrocytes (Fig. 7j), suggesting a greater involvement of microglia/macrophages compared to astrocytes in the therapeutic effect of carnosine. Given that carnosine is a multifunctional dipeptide [17] and to examine whether the acrolein 
quenching ability of carnosine alleviates neuroinflammation, we next compared the effect of the dipeptide carnosine to its constituent amino acids ( $\beta$-alanine, histidine, or both), its $\mathrm{N} \pi$ - and Nt-methylated analogues (anserine, balenine) and an equimolar negative control (tyrosyl-histidine) on inflammation-induced cellular nitrite release by microglia. Carnosine and anserine have similar carbonyl quenching properties, whereas balenine, $\beta$-alanine and histidine exhibit low carbonyl quenching ability [21,39]. As shown in Fig. $7 \mathrm{k}$, pre-treatment with the dipeptides carnosine, anserine and balenine reduced nitrite release, in contrast to individual amino acids and negative control. At a concentration of $10 \mathrm{mM}$, carnosine was significantly more effective than anserine and balenine $(\mathrm{p}<0.01)$. In summary, in vitro experiments indicate that inflammatory microglia/macrophages are a source of acrolein, which can be efficiently quenched by increasing carnosine availability, resulting in suppressed inflammatory activity. Yet, considering that acrolein quenching by balenine is minimal compared to carnosine and anserine, we cannot exclude that other properties, such as direct antioxidant activity or nitric oxide scavenging, also contribute to the versatile actions of carnosine (treatment) during neuroinflammation.

\section{Discussion}

Whilst current MS therapeutics effectively target peripheral immune responses and/or the entrance of autoreactive lymphocytes into the CNS, there is an unmet need for local neuroprotective agents able to slow down the disease progression that accompanies sustained CNS inflammation and cellular injury $[2,4]$. Acrolein, a highly reactive and toxic carbonyl, was strongly upregulated in inflammatory MS and EAE lesions, whereas the endogenous acrolein quencher carnosine was decreased in EAE. When tissue carnosine levels were increased via oral administration, acrolein-protein adduct formation and neuroinflammation were profoundly attenuated. In vivo and in vitro experiments suggest an essential role of microglia/macrophages in the generation of acrolein and the therapeutic effect of carnosine. Our findings identify carbonyl (in particular acrolein) quenching by carnosine as a therapeutic strategy to counter inflammation and macromolecular damage in MS, and possibly other neuroinflammatory disorders.

Among the variety of reactive carbonyls generated by oxidative decomposition of PUFAs, the 3-carbon $\alpha, \beta$ unsaturated aldehyde acrolein is the strongest electrophile, capable of exerting long-lasting cytotoxic changes $[6,7,40]$. Recently, an increase in urinary and serum 3-hydroxypropyl mercapturic acid (3-HPMA), a GSHacrolein metabolite, was reported in MS patients; for the first time proposing the pathological role of acrolein in MS [12]. In the present study, we have identified acrolein in active MS lesions. In EAE mice, acrolein-protein adduct formation in spinal cord appeared as a dynamic process related to disease stage and inflammatory activity. Likewise, acrolein-protein adducts in MS seemed more common in active than mixed active/inactive lesions. Acrolein damage in MS/EAE lesions occurred in a variety of structures in the brain parenchyma and perivascular spaces, often in close vicinity to microglia/ macrophages and reactive astrocytes. Together with our in vitro findings that indicate acrolein generation by pro-inflammatory microglia, this implies that the generation of acrolein, pro-inflammatory activity and the oxidative burst are entwined and may occur in a selfsustaining manner during neuroinflammation. In fact, previous studies showed that low levels of acrolein can promote inflammatory activity and ROS production without affecting cell viability [41-43]. In addition, acrolein possesses strong cytotoxic effects through binding with essential cellular macromolecules, as evidenced by the great levels of acrolein-protein adducts in the present report. Interestingly, the strong increase in acroleinprotein adducts was not accompanied by the presence of HNE-protein adducts in EAE. Although we did not

\section{(See figure on next page.)}

Fig. 6 Carnosine treatment attenuates disease burden, acrolein-protein adduct formation and markers of neuroinflammation in EAE. a Clinical EAE disease severity, scored daily (day 0-56 post immunization) by a blinded assessor, indicates a significantly lower cumulative disease burden in animals receiving $3 \%$ carnosine compared to all other groups. $n=16$ animals/group. $\mathbf{b}$ Detection of acrolein-protein adducts by western blot in healthy controls (dashed line), untreated EAE animals (0\%) and following treatment with carnosine (3\% in drinking water) in EAE. Groups receiving $0.3 \%$ and $1.5 \%$ were omitted to facilitate direct comparison of $0 \%$ and $3 \%$ carnosine on a limited number of blots. All treatment groups were compared in a preliminary experiment (data not shown). c, $\mathbf{d}$ Immunohistochemistry-based quantification of spinal cord T cell numbers (CD3) shows a reduction in carnosine-treated mice (3\%) compared to untreated mice (0\%) and mice treated with $0.3 \%$ or $1.5 \%$ carnosine in the drinking water. $\mathbf{e}, \mathbf{f}$ Immunohistochemistry-based quantification of spinal cord microglia/macrophage numbers (F4/80) shows a reduction in carnosine-treated mice (3\%) compared to untreated mice (0\%). $\mathbf{g}, \mathbf{h}$ Immunohistochemistry-based quantification of spinal cord reactive astrocyte area (GFAP) showed no treatment effect of carnosine. For figures $\mathbf{d}, \mathbf{f}$ and $\mathbf{h}$, all EAE groups exhibited a significant increase compared to healthy controls (statistics not shown). Figures $\mathbf{d}, \mathbf{f}, \mathbf{h}$ : $n=5-6$ animals/group. i Spinal cord mRNA expression in healthy controls, untreated EAE animals $(0 \%)$ and following treatment with different doses of carnosine $(0.3 \%, 1.5 \%, 3 \%$ in the drinking water) in EAE. $n=5-6$ animals/group. For all genes, untreated EAE groups (0\%) exhibited a significant increase compared to controls (statistics not shown). Data are mean \pm SD. One-way ANOVA or Kruskal-Wallis, post hoc testing ${ }^{*} p<0.05,{ }^{* *} p<0.01$ between the indicated groups $(\mathbf{a}, \mathbf{d}, \mathbf{f}, \mathbf{h}, \mathbf{i})$. Independent samples t-test ${ }^{*} p<0.05$, control line is for reference (b). Scale bars are $100 \mu \mathrm{m}(\mathbf{c}, \mathbf{e}, \mathbf{g})$ 


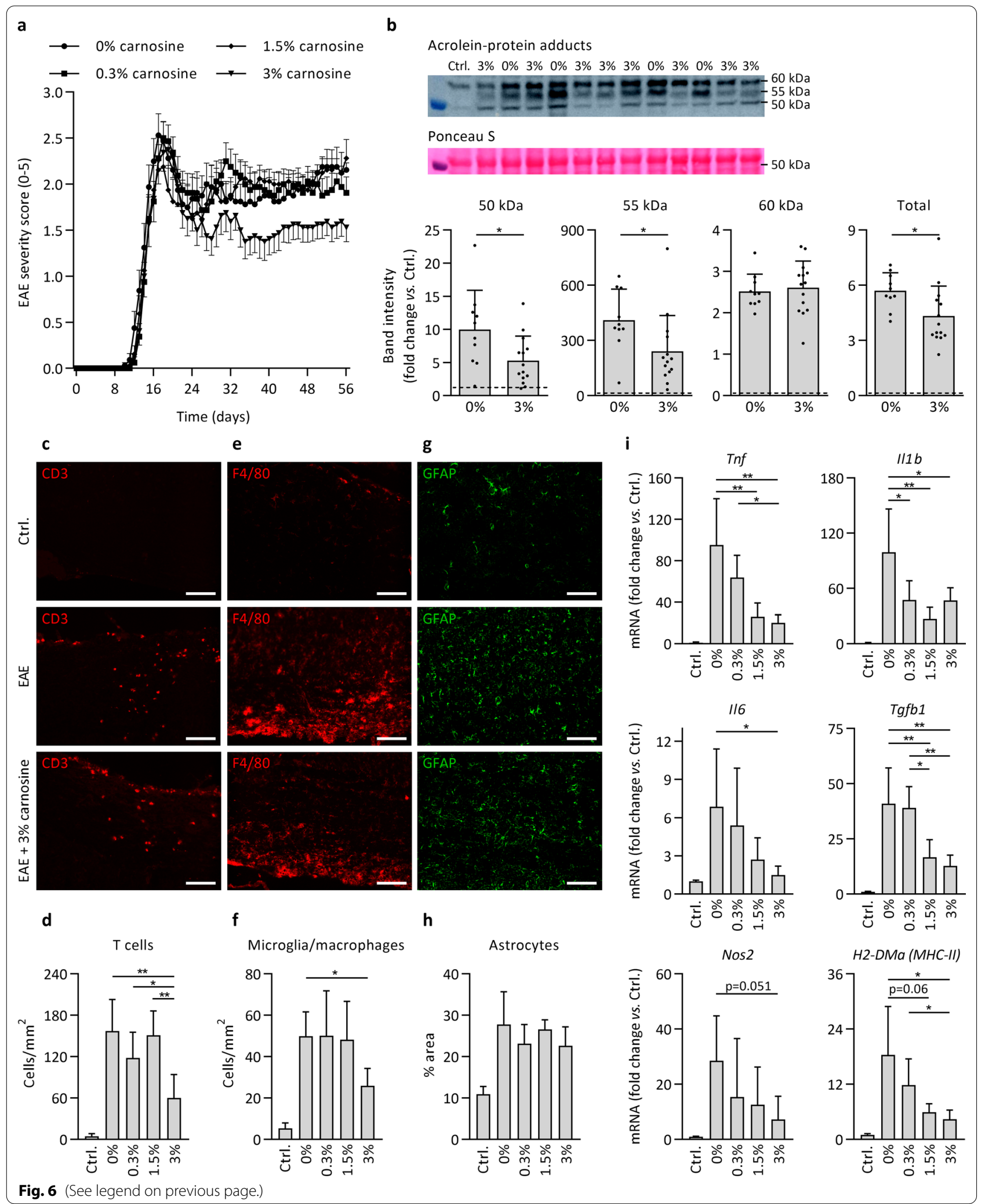


define specific molecular structures that are affected by acrolein and whether acrolein toxicity contributed directly to demyelination/oligodendropathy or axonal degeneration in MS [44], acrolein seems a valuable therapeutic target. Future research is warranted to decipher how, at what concentration, and in what disease stage(s) acrolein affects the (neuro)immune and degenerative processes that drive MS progression.

Given that reducing acrolein-protein adduct formation by carnosine was associated with attenuated disease severity in the EAE model further supports a pathological role of acrolein in MS. Carnosine quenched inflammation-induced acrolein and suppressed inflammatory and pro-oxidative activity in vitro and in vivo. Our results propose microglia/macrophages as important players in both the generation of acrolein and the therapeutic benefits of carnosine. The downstream effects of reducing acrolein levels in the CNS remain speculative but can be widespread. For example, by reducing cytokine and MHC-II expression, carnosine may indirectly affect $\mathrm{T}$ cell (re)activation or survival in the CNS [45]. A direct peripheral immunosuppressive effect of carnosine is less likely given the lack of effect at EAE disease onset and during development of the initial disease peak. It should be noted, however, that acrolein quenching may not explain all therapeutic benefits of carnosine. As can be expected from this versatile molecule, other properties including direct antioxidant or nitric oxide scavenging activity may contribute to its beneficial effects in EAE. This is particularly true given that at least some in vitro anti-inflammatory effects were retained by balenine, a methylated carnosine analogue with limited acrolein quenching ability [21,39]. Finally, it should be noted that oral carnosine treatment was given prophylactically in order to allow sufficient time for tissue carnosine loading to occur. Future research should investigate in more depth how the timing of carnosine treatment affects the results.

Carbonyl quenching is a relatively novel therapeutic approach for MS, in contrast to traditional antioxidant therapies that often had limited clinical efficacy [46-48]. Previous efforts include the use of the carbonyl quenchers pyridoxamine and hydralazine in EAE. Pyridoxamine showed no beneficial effect in EAE [49]. Compared to carnosine, pyridoxamine is a less efficient acrolein quencher but more reactive towards malondialdehyde [16], suggesting that acrolein plays an important role in EAE pathogenesis and that the quenching agent needs to be chosen accordingly. Hydralazine is a very potent carbonyl quencher that improved EAE outcomes [11], even to a greater extent than observed for carnosine in the present study. However, hydralazine is a potent hypotensive drug that lacks quenching specificity and also reacts with physiological carbonyls, making it prone to elicit undesirable side-effects [16]. Since carnosine is an endogenous and food-derived molecule that has already been applied safely in human trials $[29,50]$ and even in a recent case series with three MS patients [51], larger-scale testing of carnosine intervention in MS may advance relatively quickly. Despite carnosine's favorable toxicological profile and ability to cross the blood-brain barrier, one major disadvantage is a limited stability in human plasma due to the presence of the carnosine-degrading enzyme serum carnosinase or CN1 (not present in rodents) [17, 52]. Although the CNS possesses carnosine synthase activity to re-synthesize carnosine from $\beta$-alanine and histidine, it may be worthwhile to consider carnosinaseresistant carnosine analogues for application in humans; especially since carbonyl quenching properties of carnosine are diminished or lost by the individual amino acids. For example, the recently developed synthetic carnosine analogue carnosinol maintains physiological properties but is resistant to degradation $[8,53,54]$. The natural

\footnotetext{
(See figure on next page.)

Fig. 7 Carnosine quenches inflammation-induced acrolein and modulates microglia and macrophage inflammation in vitro. The experimental setup is shown in (a). For all experiments, cells were pre-treated with carnosine (or an alternative) for $24 \mathrm{~h}$, after which the culture medium was refreshed and cells were exposed to $L P S, H_{2} \mathrm{O}_{2}, \mathrm{PMA}$, IFN- $\gamma / \mathrm{IL}-1 \beta$ or vehicle control (PBS, represented by the horizontal dashed lines in $\left.\mathbf{d}-\mathbf{k}\right)$. $\mathbf{b}, \mathbf{c}$ UPLC-ESI-MS/MS-based quantification of carnosine and carnosine-propanal in primary mouse microglia. $n=4-5$ biological replicates/condition. Carnosine-propanal was undetectable in all experimental conditions lacking carnosine pre-treatment, in $4 / 5$ biological replicates without LPS or $\mathrm{H}_{2} \mathrm{O}_{2}$ (following carnosine), and in 1/5 biological replicates using $\mathrm{H}_{2} \mathrm{O}_{2}$ (following carnosine). Carnosine-propanal values ( 0.004 to $0.012 \mathrm{nmol} / 1 \mathrm{e} 6$ cells) were transformed $\left(\times 10^{3}\right)$ to facilitate visualisation on the $\mathrm{Y}$ axis. $\mathbf{d}$ Gene expression in primary mouse microglia. $\mathrm{n}=3$ biological replicates/ condition. e MHC-II mean fluorescence intensity (MFI) determined by flow cytometry on F4/80+ primary mouse microglia. $n=11$ biological replicates/condition. $\mathbf{f}$ Intracellular ROS levels derived from DCFH-DA fluorescence in primary mouse microglia. $n=6$ biological replicates/ condition. $\mathbf{g}$, $\mathbf{h}$ Nitrite concentration in the culture medium of primary mouse microglia following exposure to LPS or IFN- $\gamma / \mathrm{IL}-1 \beta . n=5-8$ biological replicates/condition. $\mathbf{i}$ Nitrite concentration in the culture medium of primary mouse BMDMs and $\mathbf{j}$ primary mouse astrocytes. $n=5-8$ biological replicates/condition. $\mathbf{k}$ Nitrite concentration in the culture medium of primary mouse microglia. Carnosine (Car), anserine (Ans), balenine (Bal), $\beta$-alanine ( $\beta$-ala), histidine (His), $\beta$-alanine and histidine ( $\beta$-ala/His), tyrosyl-histidine (Tyr-his). $n=5-13$ biological replicates/condition. BMDM, bone marrow-derived macrophages; DCFH-DA, 2',7'-Dichlorofluorescin diacetate; $\mathrm{H}_{2} \mathrm{O}_{2}$, hydrogen peroxide; LPS, lipopolysaccharide; MFI, mean fluorescence intensity; PBS, phosphate-buffered saline; PMA, phorbol 12-myristate 13-acetate; ROS, reactive oxygen species. Data are mean \pm SD. Mann-Whitney $U$ test with Bonferroni-adjusted $p$ values for multiple testing ${ }^{*} p<0.05(\mathbf{b}, \mathbf{c})$. Mann-Whitney $U$ test ${ }^{*} p<0.05$, ${ }^{* *} p<0.01(\mathbf{d}, \mathbf{e})$. One-way ANOVA or Kruskal-Wallis, post hoc testing ${ }^{*} p<0.05,{ }^{* *} p<0.01$ between the indicated groups $(\mathbf{f}-\mathbf{k})$
} 
a

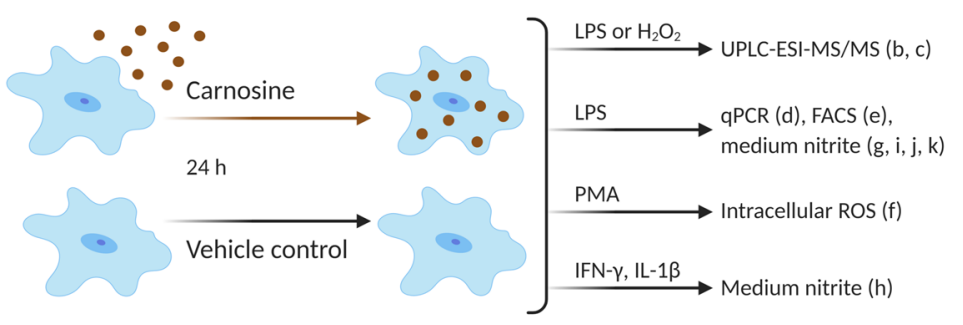

C

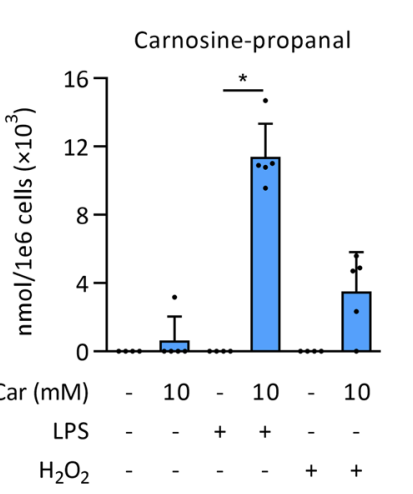

f

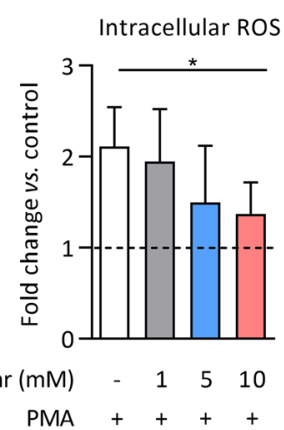

d
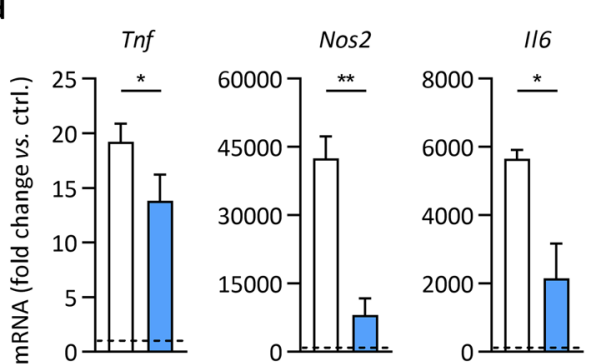

$\operatorname{Car}(\mathrm{mM}) \quad-10$

- 10

$-10$

LPS + +

$++$

$++$ b

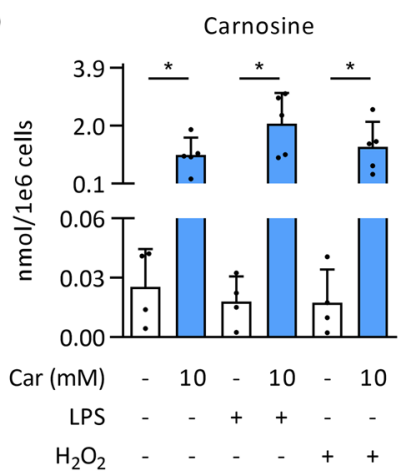

H2-DMa (MHC-II)
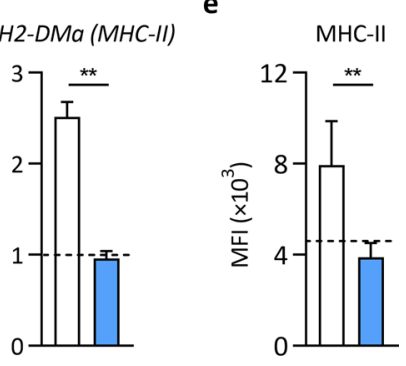

$-10$

$\operatorname{Car}(\mathrm{mM}) \quad-10$

LPS + +

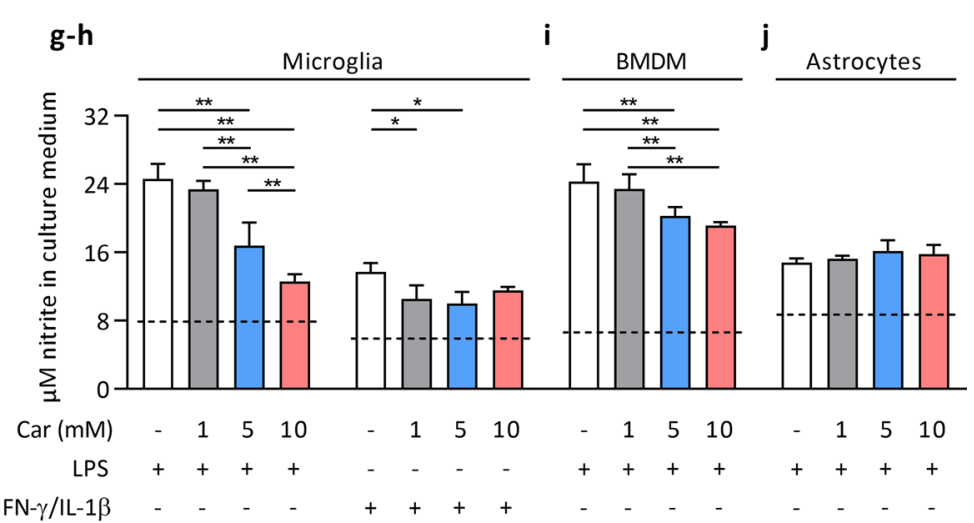

k

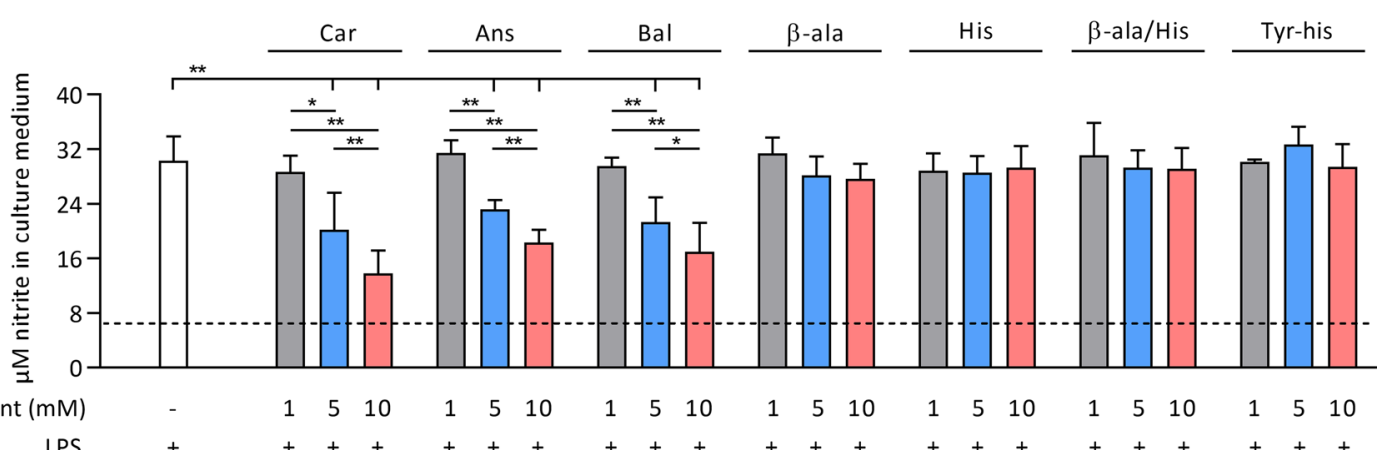

Fig. 7 (See legend on previous page.) 
carnosine analogue anserine also has better stability towards $\mathrm{CN} 1$ and retains acrolein-quenching ability [55].

Tissue carnosine homeostasis is maintained by a complex interplay between enzymatic synthesis and hydrolysis reactions, amino acid/dipeptide transport, and conjugation with reactive carbonyls such as acrolein $[17,20]$. In the present study we found that the levels of endogenous carnosine and carnosine-acrolein conjugates were depleted and the levels of acrolein-protein adducts were increased within the spinal cord of EAE mice. Given that carnosine binds with acrolein, increased sacrificial acrolein quenching and extrusion of carnosine-acrolein conjugates might contribute to the depleted carnosine pool $(-46 \%)$. Although homocarnosine (in which $\beta$-alanine is replaced by $\gamma$-aminobutyric acid [GABA]) was the most abundant HCD present in the mouse spinal cord, only carnosine showed detectable quenching activity towards acrolein, forming carnosine-propanal. In addition, mRNA data suggests that decreased synthesis (Carns1) and/or increased breakdown (Cndp1/2) could also contribute towards carnosine depletion in EAE spinal cord. In the mouse CNS, Cndp2 (as well as transporters Pht1/2, Pept2) are mainly expressed by microglia and astrocytes [56], which may explain their general increase during EAE. Human and mouse RNA sequencing studies indicate that CARNS1 is almost exclusively expressed in mature oligodendrocytes [56-63]. Oligodendrocyte dysfunction, death and demyelination may therefore underlie the reduction in Carns1 expression seen in acute EAE [64]. In contrast, by single-nucleus RNA sequencing of human brain white matter, Jäkel et al. found that CARNS1 expression was increased in active MS lesions compared to healthy controls [65]. As we found that Carns1 expression was restored in later stages of EAE, along with gradually rising tissue carnosine levels (even slightly higher than control mice), this may indicate a protective response. A similar mechanism has been suggested for muscle tissue of type 2 diabetes mellitus patients, where elevated carnosine levels presumably reflect a higher need for defence against oxidative and carbonyl stress [66]. To fully understand the significance of carnosine homeostasis in CNS health and disease, additional research is strongly recommended.

It is worth emphasizing the remarkable ability to increase carnosine levels in the mouse spinal cord upon oral carnosine intake. In fact, spinal cord seemed to have an overall greater increase in carnosine compared to muscle tissue, which has been extensively studied and is already well-known for its ability to augment carnosine content [17]. To allow direct comparison between spinal cord and muscle tissue, we assessed carnosine levels in $m$. tibialis anterior from healthy and EAE mice by UPLC-ESI-MS/MS. Compared to muscle, the spinal cord of healthy mice exhibited a greater absolute (nmol/ mgWW, $\sim 1.4$-fold greater increase) and especially relative increase $(\%, \sim$ sixfold greater increase). Data are available upon request and were consistent with previous reports of muscle carnosine loading, e.g. [67]. In EAE mice, the amount of carnosine loading was both dose- and time-dependent. The fact that carnosine loading efficiency was enhanced during the (sub)acute stages of disease, reaching carnosine levels $>$ tenfold of baseline, further highlights that the regulation of carnosine homeostasis is sensitive to disease-associated alterations. Although hypothetical, this may be explained by a local hypercellular environment featured by an abundance of dipeptide transporters on microglia/macrophages and astrocytes. In addition, it was previously shown that inflammation (LPS/IFN- $\gamma$ exposure) can stimulate carnosine uptake in RAW 264.7 macrophages by threefold [68]. Altogether, this illustrates a previously unknown capacity of the spinal cord to take up and retain carnosine, and underscores the favorable bioavailability of oral carnosine. Finally, the fact that anserine levels increased with augmented carnosine availability suggests the presence of an active protein methyltransferase enzyme, probably carnosine $N$-methyltransferase (CARNMT1); whilst the detection of carnosine-propanol in carnosine-treated EAE mice implies the reduction of carnosine-propanal by aldose reductase (AR) in spinal cord [20,69].

\section{Conclusion}

To conclude, increased acrolein generation and the resulting macromolecular damage may play a crucial pathological role during neuroinflammation in MS and EAE. This was associated with depletion of carnosine, an endogenous acrolein quencher. Oral carnosine treatment prevented acrolein damage in EAE spinal cord and attenuated neuroinflammation and diseases severity. Our findings pave the way for future research focusing on the effect of carbonyl (in particular acrolein) quenching in neuroinflammatory disorders such as MS. In addition, we highlight the need for a better understanding of endogenous carnosine homeostasis in the CNS.

\footnotetext{
Abbreviations

AUC: Area under the curve; BMDM: Bone marrow-derived macrophages; CN1: Serum carnosinase; CNS: Central nervous system; DAB: 3,3'-Diaminobenzidine; DCFH-DA: 2',7'-Dichlorofluorescin diacetate; EAE: Experimental autoimmune encephalomyelitis; FVD: Fixable viability dye; GFAP: Glial fibrillary acidic protein; GSH: Glutathione; $\mathrm{H}_{2} \mathrm{O}_{2}$ : Hydrogen peroxide; $\mathrm{HCD}$ : Histidine-containing dipeptide; HNE: 4-Hydroxy-2-nonenal; LCM: L929 cell-conditioned medium; LPS: Lipopolysaccharide; MS: Multiple sclerosis; PBS: Phosphate-buffered saline; PLP: Proteolipid protein; PMA: Phorbol 12-myristate 13-acetate; PUFA: Poly-unsaturated fatty acids; ROS: Reactive oxygen species; UPLC-ESI-MS/MS: Ultrahigh-performance liquid chromatography electrospray ionization tandem mass spectrometry; WW: Wet tissue weight.
} 


\section{Supplementary Information}

The online version contains supplementary material available at https://doi. org/10.1186/s12974-021-02306-9.

Additional file 1: Table S1. Clinical details of human brain tissue.

Additional file 2: Table S2. Oral carnosine treatment attenuates clinical disability in a monophasic rat EAE model.

Additional file 3: Table S3. Primer sequences.

Additional file 4: Figure S1. Graphical summary of experimental designs. Graphical representation of the timing of carnosine treatment, EAE induction and sacrifice (tissue collection) for (a) analyses of control vs. EAE mice (data shown in Figure 2, Figure 3, Figure S2, Figure S3, Figure S4); (b) analysis of tissue carnosine loading in EAE (data shown in Figure 4); and (c) analyses of dose-dependent carnosine treatment effects (data shown in Figure 5, Figure 6, Figure S5, Figure S6). Figure S2. Spinal cord inflammatory activity during EAE. (a) Spinal cord mRNA expression in different stages of EAE compared to healthy controls (dashed line). $\mathrm{n}=6$ animals/group. (b) Immunohistochemistry-based quantification of $\mathrm{F} 4 / 80^{+}$microglia/macrophage numbers in the spinal cord in different stages of EAE compared to healthy controls (dashed line). $n=6$ animals/ group. Gene expression (a) and microglia/macrophage numbers (b) were increased in EAE vs. healthy controls (statistics not shown). Data are mean \pm SD. One-way ANOVA or Kruskal-Wallis, post hoc testing ${ }^{*} p<0.05,{ }^{* *} p<0.01$ between the indicated groups. Figure S3. HNE-protein adducts are not affected by EAE. Detection of HNE-protein adducts by western blot in different stages of EAE compared to healthy controls. $\mathrm{n}=5-7$ animals/group. Data are mean \pm SD. One-way ANOVA indicated no significant between-group differences. Figure S4. Spinal cord GSH levels in EAE. UPLC-ESI-MS/MS-based quantification of spinal cord GSH in different stages of EAE compared to healthy controls. $n=11-22$ animals/ group. GSH, reduced glutathione. Data are mean $\pm \mathrm{SD}$. One-way ANOVA, post hoc testing ${ }^{*} p<0.05,{ }^{* *} p<0.01$ between the indicated groups. Figure S5. Effects of carnosine treatment on homocarnosine, anserine and GSH levels in chronic EAE. UPLC-ESI-MS/MS-based quantification of spinal cord and plasma $(a, d)$ homocarnosine, $(b, e)$ anserine and $(c, f)$ GSH in healthy controls, untreated EAE animals (0\%) and following treatment with different doses of carnosine $(0.3 \%, 1.5 \%, 3 \%)$ in EAE. $n=7-11$ animals/group. Data are mean \pm SD. One-way ANOVA or Kruskal-Wallis, post hoc testing ${ }^{*} p<0.05,{ }^{* *} p<0.01$ between the indicated groups. Figure S6. No effect of EAE and carnosine treatment on HNE-protein adduct levels. Detection of HNE-protein adducts by western blot in healthy controls, untreated EAE animals (0\%) and following treatment with different doses of carnosine $(0.3 \%, 1.5 \%, 3 \%$ in drinking water $)$ in EAE. $n=7-15$ animals/group. Data are mean \pm SD. One-way ANOVA indicated no significant between-group differences. Figure S7. Effect of carnosine on acrolein quenching, anserine levels and cell viability in primary mouse microglia cultures. UPLC-ESI-MS/ MS-based quantification of (a) carnosine and carnosine-propanal and (b) anserine in primary mouse microglia exposed to carnosine or vehicle control (PBS) for $24 \mathrm{~h}$, followed by LPS, $\mathrm{H}_{2} \mathrm{O}_{2}$ or vehicle control (PBS) for $24 \mathrm{~h}$. $\mathrm{n}=4-5$ biological replicates/condition. Carnosine-propanal values $(\sim 0.004$ to $0.012 \mathrm{nmol} / 1$ e6 cells) were transformed $\left(\times 10^{3}\right)$ to facilitate visualisation on the $Y$ axis. Anserine was undetectable in all experimental conditions lacking carnosine pre-treatment. (c) Cell viability of primary microglia assessed by flow cytometry (Fixable Viability Dye eFluor 506). Cells were exposed to carnosine or vehicle control (PBS) for $24 \mathrm{~h}$, followed by LPS, $\mathrm{H}_{2} \mathrm{O}_{2}$ or vehicle control (PBS) for $24 \mathrm{~h}$. Data from $\mathrm{n}=7$ different experiments, normalized to the respective control condition in each experiment and expressed as \% change. Car, carnosine. $\mathrm{H}_{2} \mathrm{O}_{2}$, hydrogen peroxide; LPS, lipopolysaccharide; PBS, phosphate-buffered saline. Data are mean \pm SD. Mann-Whitney $U$ test with Bonferroni-adjusted $p$ values for multiple testing ${ }^{*} p<0.05$ (b). One-way ANOVA indicated no significant between-group differences (c).

\section{Acknowledgements}

We sincerely thank prof. dr. Aruni Bhatnagar from the University of Louisville, Diabetes and Obesity Center for his hospitality and useful advice. We are grateful to Flamma (Flamma Group, Chignolo d'Isola, Bergamo, Italy) for providing L-carnosine and L-anserine, and K. Wauterickx (Hasselt University) for assistance with immunohistochemistry on human brain samples. Figure $7 \mathrm{a}$ was created in BioRender.

\section{Authors' contributions}

$J S, W D$ and $B O E$ led the conceptualisation and design of experiments (in collaboration with TV, JB, BB, NH, JVH and SPB). JS, WMFA, CK, LB, DKP and DH conducted the experiments. JS and $\mathrm{DH}$ acquired the data. JS analysed the data. JS, WD and BOE drafted the initial version of the manuscript, which was thoroughly reviewed and approved by all authors in its final form.

\section{Funding}

This research was funded by the Special Research Fund (BOF, Hasselt University, Belgium) and Research Foundation Flanders (FWO Vlaanderen, Belgium; $1138520 \mathrm{~N})$.

\section{Availability of data and materials}

The datasets used and/or analysed during the current study are available through the corresponding author on reasonable request.

\section{Declarations}

\section{Ethics approval and consent to participate}

The human brain samples were obtained from The Netherlands Brain Bank (NBB), Netherlands Institute for Neuroscience, Amsterdam (open access www. brainbank.nl). All material has been collected from donors for or from whom a written informed consent for a brain autopsy and the use of the material and clinical information for research purposes had been obtained by the NBB. The experiment protocols and methods used for analysing brain samples were conducted with the approval of the NBB and the Medical Ethical Committee of Hasselt University, and carried out according to institutional guidelines. Animal study procedures were approved by the Hasselt University Animal Ethics Committee and complied with national/European legislation and the National Research Council's guide for the care and use of laboratory animals.

\section{Consent for publication}

Not applicable.

\section{Competing interests}

The authors declare that they have no competing interests.

\section{Author details}

${ }^{1}$ University MS Center (UMSC) Hasselt - Pelt, Hasselt, Belgium. ${ }^{2}$ BIOMED Biomedical Research Institute, Faculty of Medicine and Life Sciences, Hasselt University, Hasselt, Belgium. ${ }^{3}$ Department of Movement and Sports Sciences, Faculty of Medicine and Health Sciences, Ghent University, Ghent, Belgium. ${ }^{4}$ REVAL Rehabilitation Research Center, Faculty of Rehabilitation Sciences, Hasselt University, Hasselt, Belgium. ${ }^{5}$ Neuro-Immune Connections and Repair Lab, Department of Immunology and Infection, Biomedical Research Institute, Hasselt University, Diepenbeek, Belgium. ${ }^{6}$ Division of Translational Neuroscience, Department Psychiatry and Neuropsychology, European Graduate School of Neuroscience, School for Mental Health and Neuroscience, Maastricht University, Maastricht, The Netherlands. ${ }^{7}$ Department of Internal Medicine, Cardiovascular Research Institute Maastricht, Maastricht University, Maastricht, The Netherlands. ${ }^{8}$ Department of Molecular Cell Biology and Immunology, Amsterdam Neuroscience, MS Center Amsterdam, Amsterdam University Medical Center, Location VUmc, Amsterdam, The Netherlands. ${ }^{9}$ Diabetes and Obesity Center, University of Louisville, Louisville, KY, USA.

Received: 15 April 2021 Accepted: 22 October 2021

Published online: 05 November 2021

\section{References}

1. Dendrou CA, Fugger L, Friese MA. Immunopathology of multiple sclerosis. Nat Rev Immunol. 2015;15(9):545-58.

2. Baecher-Allan C, Kaskow BJ, Weiner HL. Multiple sclerosis: mechanisms and immunotherapy. Neuron. 2018;97(4):742-68. 
3. Bogie JF, Stinissen P, Hendriks JJ. Macrophage subsets and microglia in multiple sclerosis. Acta Neuropathol. 2014;128(2):191-213.

4. Ontaneda D, Thompson AJ, Fox RJ, Cohen JA. Progressive multiple sclerosis: prospects for disease therapy, repair, and restoration of function. Lancet. 2017;389(10076):1357-66.

5. Witte ME, Geurts JJ, de Vries HE, van der Valk P, van Horssen J. Mitochondrial dysfunction: a potential link between neuroinflammation and neurodegeneration? Mitochondrion. 2010;10(5):411-8.

6. Aldini G, Orioli M, Carini M. Protein modification by acrolein: relevance to pathological conditions and inhibition by aldehyde sequestering agents. Mol Nutr Food Res. 2011;55(9):1301-19.

7. Sousa BC, Pitt AR, Spickett CM. Chemistry and analysis of HNE and other prominent carbonyl-containing lipid oxidation compounds. Free Radical Biol Med. 2017;111:294-308.

8. Anderson EJ, Vistoli G, Katunga LA, Funai K, Regazzoni L, Monroe TB, et al. A carnosine analog mitigates metabolic disorders of obesity by reducing carbonyl stress. J Clin Investig. 2018;1 28(12):5280-93.

9. Haus JM, Thyfault JP. Therapeutic potential of carbonyl-scavenging carnosine derivative in metabolic disorders. J Clin Investig. 2018:128(12):5198-200.

10. Haider L, Fischer MT, Frischer JM, Bauer J, Höftberger R, Botond $\mathrm{G}$, et al. Oxidative damage in multiple sclerosis lesions. Brain. 2011;134(7):1914-24

11. Leung G, Sun W, Zheng L, Brookes S, Tully M, Shi R. Anti-acrolein treatment improves behavioral outcome and alleviates myelin damage in experimental autoimmune enchephalomyelitis mouse. Neuroscience. 2011;173:150-5.

12. Tully M, Tang J, Zheng L, Acosta G, Tian R, Hayward L, et al. Systemic acrolein elevations in mice with experimental autoimmune encephalomyelitis and patients with multiple sclerosis. Front Neurol. 2018;9:420.

13. Dunham J, Bauer J, Campbell GR, Mahad DJ, van Driel N, van der Pol S, et al. Oxidative injury and iron redistribution are pathological hallmarks of marmoset experimental autoimmune encephalomyelitis. J Neuropathol Exp Neurol. 2017;76(6):467-78.

14. Bizzozero OA, DeJesus G, Callahan K, Pastuszyn A. Elevated protein carbonylation in the brain white matter and gray matter of patients with multiple sclerosis. J Neurosci Res. 2005;81(5):687-95.

15. Van Horssen J, Schreibelt G, Drexhage J, Hazes T, Dijkstra C, Van der Valk $P$, et al. Severe oxidative damage in multiple sclerosis lesions coincides with enhanced antioxidant enzyme expression. Free Radic Biol Med. 2008:45(12):1729-37.

16. Colzani M, De Maddis D, Casali G, Carini M, Vistoli G, Aldini G. Reactivity, selectivity, and reaction mechanisms of aminoguanidine, hydralazine, pyridoxamine, and carnosine as sequestering agents of reactive carbonyl species: a comparative study. ChemMedChem. 2016;11(16):1778-89.

17. Boldyrev AA, Aldini G, Derave W. Physiology and pathophysiology of carnosine. Physiol Rev. 2013;93(4):1803-45.

18. Xie Z, Baba SP, Sweeney BR, Barski OA. Detoxification of aldehydes by histidine-containing dipeptides: from chemistry to clinical implications. Chem Biol Interact. 2013;202(1-3):288-97.

19. Drozak J, Veiga-da-Cunha M, Vertommen D, Stroobant V, Van Schaftingen E. Molecular identification of carnosine synthase as ATP-grasp domaincontaining protein 1 (ATPGD1). J Biol Chem. 2010;285(13):9346-56.

20. Baba SP, Hoetker JD, Merchant M, Klein JB, Cai J, Barski OA, et al. Role of aldose reductase in the metabolism and detoxification of carnosineacrolein conjugates. J Biol Chem. 2013;288(39):28163-79.

21. Zhao J, Posa DK, Kumar V, Hoetker D, Kumar A, Ganesan S, et al. Carnosine protects cardiac myocytes against lipid peroxidation products. Amino Acids. 2019;51(1):123-38.

22. Carini M, Aldini G, Beretta G, Arlandini E, Facino RM. Acrolein-sequestering ability of endogenous dipeptides: characterization of carnosine and homocarnosine/acrolein adducts by electrospray ionization tandem mass spectrometry. J Mass Spectrom. 2003;38(9):996-1006.

23. Esterbauer H, Schaur RJ, Zollner H. Chemistry and biochemistry of 4-hydroxynonenal, malonaldehyde and related aldehydes. Free Radic Biol Med. 1991:11(1):81-128.

24. Vistoli G, Colzani M, Mazzolari A, Maddis DD, Grazioso G, Pedretti A, et al. Computational approaches in the rational design of improved carbonyl quenchers: focus on histidine containing dipeptides. Future Med Chem. 2016:8(14):1721-37.
25. Vistoli G, Colzani M, Mazzolari A, Gilardoni E, Rivaletto C, Carini M, et al. Quenching activity of carnosine derivatives towards reactive carbonyl species: focus on a-(methylglyoxal) and $\beta$-(malondialdehyde) dicarbonyls. Biochem Biophys Res Commun. 2017;492(3):487-92.

26. Aldini G, Orioli M, Rossoni G, Savi F, Braidotti P, Vistoli G, et al. The carbonyl scavenger carnosine ameliorates dyslipidaemia and renal function in Zucker obese rats. J Cell Mol Med. 2011:15(6):1339-54.

27. Yamashita S, Sato M, Matsumoto T, Kadooka K, Hasegawa T, Fujimura T. et al. Mechanisms of carnosine-induced activation of neuronal cells. Biosci Biotechnol Biochem. 2018;82(4):683-8.

28. Bae O-N, Serfozo K, Baek S-H, Lee KY, Dorrance A, Rumbeiha W, et al. Safety and efficacy evaluation of carnosine, an endogenous neuroprotective agent for ischemic stroke. Stroke. 2013;44(1):205-12.

29. Regazzoni L, De Courten B, Garzon D, Altomare A, Marinello C, Jakubova $M$, et al. A carnosine intervention study in overweight human volunteers: bioavailability and reactive carbonyl species sequestering effect. Sci Rep. 2016;6:27224

30. Kuhlmann T, Ludwin S, Prat A, Antel J, Brück W, Lassmann H. An updated histological classification system for multiple sclerosis lesions. Acta Neuropathol. 2017:133(1):13-24.

31. Keytsman C, Blancquaert L, Wens I, Missine M, Noten PV, Vandenabeele F, et al. Muscle carnosine in experimental autoimmune encephalomyelitis and multiple sclerosis. Multiple Sclerosis Related Disorders. 2018;21:24-9.

32. Chesher D. Evaluating assay precision. Clin Biochem Rev. 2008;29(Suppl 1):S23.

33. Vandesompele J, De Preter K, Pattyn F, Poppe B, Van Roy N, De Paepe A, et al. Accurate normalization of real-time quantitative RT-PCR data by geometric averaging of multiple internal control genes. Genome Biol. 2002:3(7):research0034.

34. Bogie JF, Grajchen E, Wouters E, Corrales AG, Dierckx T, Vanherle S, et al. Stearoyl-CoA desaturase-1 impairs the reparative properties of macrophages and microglia in the brain. J Exp Med. 2020;217(5):1.

35. Bogie JF, Mailleux J, Wouters E, Jorissen W, Grajchen E, Vanmol J, et al. Scavenger receptor collectin placenta 1 is a novel receptor involved in the uptake of myelin by phagocytes. Sci Rep. 2017;7:44794.

36. Constantinescu CS, Farooqi N, O'brien K, Gran B. Experimental autoimmune encephalomyelitis (EAE) as a model for multiple sclerosis (MS). Br J Pharmacol. 2011:164(4):1079-106.

37. Aldini G, Orioli M, Carini M, Maffei FR. Profiling histidine-containing dipeptides in rat tissues by liquid chromatography/electrospray ionization tandem mass spectrometry. J Mass Spectrom. 2004;39(12):1417-28.

38. Choi I-Y, Lee P, Hughes AJ, Denney DR, Lynch SG. Longitudinal changes of cerebral glutathione (GSH) levels associated with the clinical course of disease progression in patients with secondary progressive multiple sclerosis. Mult Scler J. 2017;23(7):956-62.

39. Mori A, Hatate H, Tanaka R. Ability of three kind of imidazole dipeptides, carnosine, anserine, and balenine, to interact with unsaturated fatty acidderived aldehydes and carbohydrate-derived aldehydes. Int J Peptide Res Therap. 2019;19:1-10.

40. Mello C, Sultana R, Piroddi M, Cai J, Pierce W, Klein J, et al. Acrolein induces selective protein carbonylation in synaptosomes. Neuroscience. 2007;147(3):674-9.

41. Song J-J, Lee JD, Lee BD, Chae SW, Park MK. Effect of acrolein, a hazardous air pollutant in smoke, on human middle ear epithelial cells. Int J Pediatr Otorhinolaryngol. 2013;77(10):1659-64.

42. Ansari MA, Keller JN, Scheff SW. Protective effect of Pycnogenol in human neuroblastoma SH-SY5Y cells following acrolein-induced cytotoxicity. Free Radic Biol Med. 2008:45(11):1510-9.

43. Luo J, Robinson JP, Shi R. Acrolein-induced cell death in PC12 cells: role of mitochondria-mediated oxidative stress. Neurochem Int. 2005;47(7):449-57.

44. Shi Y, Sun W, McBride JJ, Cheng JX, Shi R. Acrolein induces myelin damage in mammalian spinal cord. J Neurochem. 2011;117(3):554-64.

45. Schetters ST, Gomez-Nicola D, Garcia-Vallejo JJ, Van Kooyk Y Neuroinflammation: microglia and T cells get ready to tango. Front Immunol. 2018:8:1905.

46. Neves Carvalho A, Firuzi O, Joao Gama M, van Horssen J, Saso L. Oxidative stress and antioxidants in neurological diseases: is there still hope? Curr Drug Targets. 2017;18(6):705-18. 
47. Gilgun-Sherki Y, Melamed E, Offen D. The role of oxidative stress in the pathogenesis of multiple sclerosis: the need for effective antioxidant therapy. J Neurol. 2004;251(3):261-8.

48. Miller ED, Dziedzic A, Saluk-Bijak J, Bijak M. A review of various antioxidant compounds and their potential utility as complementary therapy in multiple sclerosis. Nutrients. 2019;11(7):1528.

49. Wetzels S, Wouters K, Miyata T, Scheijen JL, Hendriks JJ, Schalkwijk CG, et al. Advanced glycation endproducts are increased in the animal model of multiple sclerosis but cannot be reduced by pyridoxamine treatment or glyoxalase 1 overexpression. Int J Mol Sci. 2018;19(5):1311.

50. Boldyrev A, Fedorova T, Stepanova M, Dobrotvorskaya I, Kozlova E, Boldanova N, et al. Carnisone increases efficiency of DOPA therapy of Parkinson's disease: a pilot study. Rejuvenation Res. 2008;11(4):821-7.

51. Zanini D, Jezdimirovic T, Stajer V, Ostojic J, Maksimovic N, Ostojic SM. Dietary supplementation with L-carnosine improves patient-reported outcomes, autonomic nervous system performance, and brain metabolism in three adult patients with multiple sclerosis. Nutr Res. 2020;84:63-9.

52. Everaert I, Taes Y, De Heer E, Baelde H, Zutinic A, Yard B, et al. Low plasma carnosinase activity promotes carnosinemia after carnosine ingestion in humans. Am J Physiol-Renal Physiol. 2012;302:1537.

53. lacobini C, Menini S, Blasetti Fantauzzi C, Pesce CM, Giaccari A, Salomone E, et al. FL-926-16, a novel bioavailable carnosinase-resistant carnosine derivative, prevents onset and stops progression of diabetic nephropathy in db/db mice. Br J Pharmacol. 2018;175(1):53-66.

54. Menini S, lacobini C, de Latouliere L, Manni I, Vitale M, Pilozzi E, et al. Diabetes promotes invasive pancreatic cancer by increasing systemic and tumour carbonyl stress in Kras G12D/+ mice. J Exp Clin Cancer Res. 2020;39(1):1-16.

55. Everaert I, Baron G, Barbaresi S, Gilardoni E, Coppa C, Carini M, et al. Development and validation of a sensitive LC-MS/MS assay for the quantification of anserine in human plasma and urine and its application to pharmacokinetic study. Amino Acids. 2018;51:1-12.

56. Zhang Y, Chen K, Sloan SA, Bennett ML, Scholze AR, O'Keeffe S, et al. An RNA-sequencing transcriptome and splicing database of glia, neurons, and vascular cells of the cerebral cortex. J Neurosci. 2014;34(36):11929-47.

57. Zhang Y, Sloan SA, Clarke LE, Caneda C, Plaza CA, Blumenthal PD, et al Purification and characterization of progenitor and mature human astrocytes reveals transcriptional and functional differences with mouse. Neuron. 2016;89(1):37-53.

58. Dusart P, Hallström BM, Renne T, Odeberg J, Uhlén M, Butler LM. A systems-based map of human brain cell-type enriched genes and malignancy-associated endothelial changes. Cell Rep. 2019;29(6):1690-706.

59. Darmanis S, Sloan SA, Zhang Y, Enge M, Caneda C, Shuer LM, et al. A survey of human brain transcriptome diversity at the single cell level. Proc Natl Acad Sci. 2015;112(23):7285-90.

60. Saunders A, Macosko EZ, Wysoker A, Goldman M, Krienen FM, de Rivera $\mathrm{H}$, et al. Molecular diversity and specializations among the cells of the adult mouse brain. Cell. 2018;174(4):1015-30.

61. Vanlandewijck M, He L, Mäe MA, Andrae J, Ando K, Del Gaudio F, et al. A molecular atlas of cell types and zonation in the brain vasculature. Nature. 2018;554(7693):475-80.

62. Zeisel A, Hochgerner H, Lönnerberg P, Johnsson A, Memic F, Van Der Zwan J, et al. Molecular architecture of the mouse nervous system. Cell. 2018;174(4):999-1014.

63. Reddy AS, O'Brien D, Pisat N, Weichselbaum CT, Sakers K, Lisci M, et al. A comprehensive analysis of cell type-specific nuclear RNA from neurons and glia of the brain. Biol Psychiat. 2017;81(3):252-64.

64. Reynolds R, Dawson M, Papadopoulos D, Polito A, di Bello IC, Pham-Dinh $D$, et al. The response of NG2-expressing oligodendrocyte progenitors to demyelination in MOG-EAE and MS. J Neurocytol. 2002;31(6-7):523-36.

65. Jäkel S, Agirre E, Falcão AM, Van Bruggen D, Lee KW, Knuesel I, et al. Altered human oligodendrocyte heterogeneity in multiple sclerosis. Nature. 2019;566(7745):543-7.

66. Stegen S, Everaert I, Deldicque L, Vallova S, de Courten B, Ukropcova B, et al. Muscle histidine-containing dipeptides are elevated by glucose intolerance in both rodents and men. PLOS ONE. 2015;10(3):e0121062.

67. Everaert I, Stegen S, Vanheel B, Taes Y, Derave W. Effect of beta-alanine and carnosine supplementation on muscle contractility in mice. Med Sci Sports Exerc. 2013;45(1):43-51.

68. Fresta CG, Hogard ML, Caruso G, Costa EEM, Lazzarino G, Lunte SM. Monitoring carnosine uptake by RAW 264.7 macrophage cells using microchip electrophoresis with fluorescence detection. Anal Methods. 2017;9(3):402-8.

69. Drozak J, Piecuch M, Poleszak O, Kozlowski P, Chrobok L, Baelde HJ, et al. UPF0586 protein C9orf41 homolog is anserine-producing methyltransferase. J Biol Chem. 2015;290(28):17190-205.

\section{Publisher's Note}

Springer Nature remains neutral with regard to jurisdictional claims in published maps and institutional affiliations.
Ready to submit your research? Choose BMC and benefit from:

- fast, convenient online submission

- thorough peer review by experienced researchers in your field

- rapid publication on acceptance

- support for research data, including large and complex data types

- gold Open Access which fosters wider collaboration and increased citations

- maximum visibility for your research: over 100M website views per year

At BMC, research is always in progress.

Learn more biomedcentral.com/submissions 